\title{
Flight Safety Assessment and Management to Prevent Loss of Control Due to In-Flight Icing
}

\author{
Sweewarman Balachandran*and Ella M. Atkins ${ }^{\dagger}$ \\ University of Michigan, Ann Arbor, MI, 48109, USA
}

\begin{abstract}
Flight Safety Assessment and Management is a high-level decision making system that makes resilient control override decisions to prevent/recover from loss of control situations. In-flight icing is a major threat to commercial and general aviation aircraft. Icing results in envelope degradation and poor controllability that can both lead to loss of control. This paper describes the development of a Flight Safety Assessment and Management system that can identify situations with high loss of control risk due to in-flight icing and override the nominal crew-FMS system with an envelope-aware control authority that can maneuver the aircraft safely away from icing. Flight Safety Assessment and Management is formulated as a Markov Decision Process to account for uncertainties in state evolution and tradeoffs between passively monitoring crew commands versus executing a safety-based resilient control override. A Markov Decision Process policy for an in-flight icing case study is developed and evaluated.
\end{abstract}

\section{Nomenclature}

$\begin{array}{ll}\text { Acronyms } & \\ \text { EA-FMS } & \text { Envelope-Aware Flight Management System } \\ \text { FMS } & \text { Flight Management System } \\ \text { FSAM } & \text { Flight Safety Assessment and Management } \\ \text { LOC } & \text { Loss of Control } \\ \text { MDP } & \text { Markov Decision Process } \\ \text { Symbols } & \\ (\mathcal{S}, \mathcal{A}, \mathcal{T}, \mathcal{R}) & \text { MDP states, actions, transition probabilities, and rewards } \\ \bar{V}, \bar{A}, \bar{\Theta}, \bar{\Phi} & \text { Airspeed, angle of attack/sideslip, dynamic pitch and roll features } \\ \bar{v}, \bar{\alpha}, \bar{\theta}, \bar{\phi} & \text { Airspeed, angle of attack/sidelip, dynamic pitch and dynamic roll state } \\ \bar{H}, \bar{T} & \text { Vertical speed and thrust features } \\ \bar{h}, \bar{t} & \text { Vertical speed and thrust state } \\ \bar{F} & \text { Flight plan feature } \\ \bar{f} & \text { Flight plan state } \\ \bar{I} & \text { Icing severity feature } \\ \bar{i} & \text { Icing severity state } \\ \bar{M}, \bar{S} & \text { Mode, mode selector features } \\ P, E A & \text { Pilot, Envelope-Aware control modes } \\ P_{s}, E A_{s} & \text { Mode select switch status: Pilot, Envelope-Aware } \\ N O O P, T O G L & \text { No operation, Toggle MDP actions } \\ & \end{array}$

\section{Introduction}

Fly-by-wire technology has facilitated incorporation of decision aids that reduce crew workload and improve situational awareness. However, aviation accidents still occur. A majority of aviation accidents involve Loss of Control (LOC) induced by some combination of inappropriate crew response, severe winds/weather, and collision

\footnotetext{
* Student Member, Department of Aerospace Engineering, swee@umich.edu

${ }^{\dagger}$ Associate Fellow, Department of Aerospace Engineering, ematkins@umich.edu
} 
with other aircraft or terrain. ${ }^{1}$ Icing-related LOC situations are some of the most difficult cases to model and manage. Flight through atmospheric conditions conducive to icing can lead to accumulation of ice on the wings, tail surfaces and fuselage. Engine icing can also cause damage and even loss of thrust. Ice accumulation alters the shape of the airframe and disrupts airflow over the aircraft resulting in changes to its aerodynamic properties. ${ }^{2}$ Consequently, ice accumulation increases weight and drag while decreasing lift. Wing icing also leads to a decrease in the stall angle of attack, while ice contamination of the horizontal stabilizers can result in tail plane stall. ${ }^{3}$ Icing can also result in blockage of the pitot probes leading to erroneous airspeed measurements. ${ }^{4}$

Several strategies can mitigate LOC risk due to icing. Prior to departure, de-icing fluids can be sprayed over the airframe to hinder ice accumulation. Aircraft anti-icing (e.g., wing heat) and de-icing (e.g., wing boot) systems can reduce or eliminate wing surface icing during flight. Current autopilot systems are equipped with warnings and envelope protection features that can help prevent stall. ${ }^{4,5}$ However, envelope protection logic is based on the nominal performance values such as a constant critical stall angle of attack based on clean surface conditions. With wing icing, the critical angle of attack decreases with no estimate of degraded stall angle of attack provided to the pilot or autopilot. This renders the stall protection system ineffective for icing scenarios. Furthermore, the increase in drag due to icing requires the airplane to fly at higher airspeeds to produce the lift necessary to lift the aircraft in steady flight. Asymmetric ice accumulation can lead to upsets in roll control. Control surface effectiveness is also reduced due to icing. To prevent icing-related LOC, an ice protection system must first identify the changes in aerodynamics, performance and control characteristics of the aircraft due to icing then ensure that automation and ultimately crew warning systems incorporate these changes.

The Envelope Aware Flight Management System (EA-FMS) was proposed in previous publications to enable the prevention/recovery of LOC scenarios. EA-FMS consists of Flight Safety Assessment and Management, ${ }^{6-8}$ Envelope Estimation, ${ }^{9,10}$ Adaptive Flight Planning, ${ }^{11,12}$ System Identification ${ }^{13-15}$ and Adaptive Control. ${ }^{16,17}$

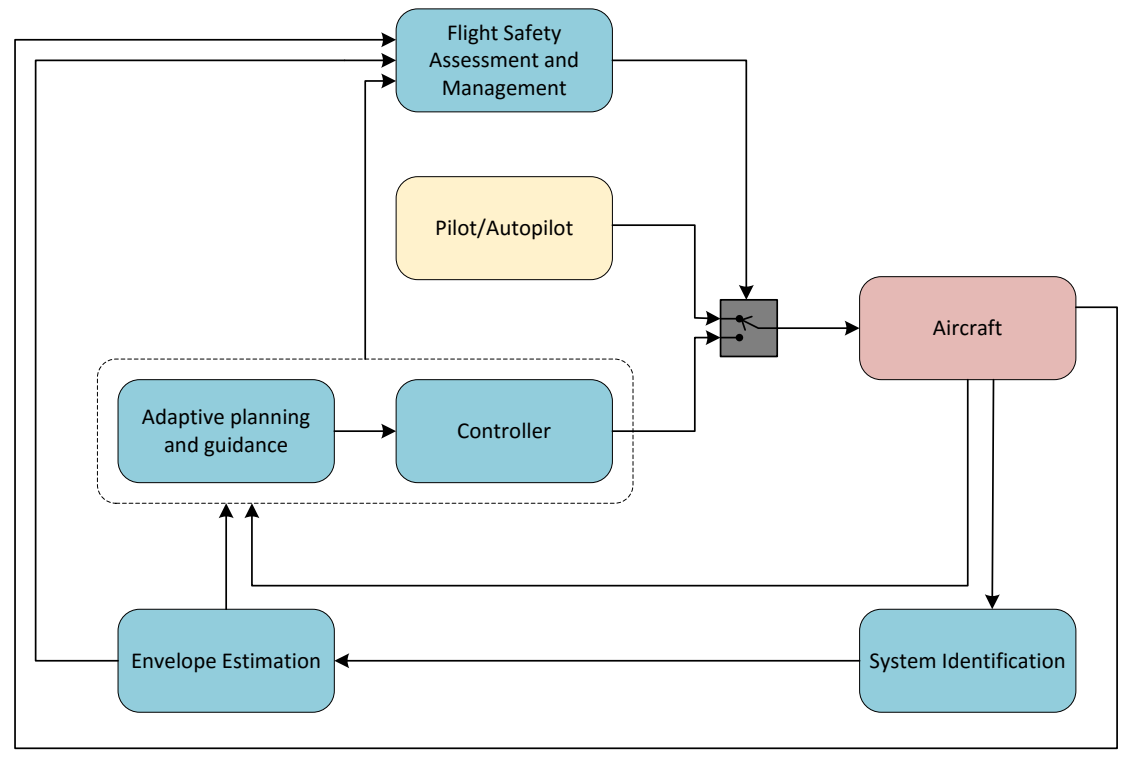

Figure 1. Envelope-Aware Flight Management (EA-FMS) system architecture

FSAM has previously been developed for high-risk LOC situations in which performance models are unchanged ${ }^{8}$ or when a one-time performance reduction occurs. ${ }^{18}$ This paper focuses on developing an FSAM capability to ensure that an appropriate control authority is chosen to prevent or recover from icing, a potentially high-risk LOC scenario where performance can degrade progressively in flight. FSAM constantly monitors flight conditions to assess LOC risk, initially warning the flight crew when LOC risk exceeds a nominal threshold. If the crew does not respond with appropriate control actions in time to assure recovery, FSAM overrides with an Envelope Aware control law from EAFMS until the LOC risk is mitigated. FSAM is effectively a "watchdog" system providing LOC avoidance override for flight envelope protection in a general context. This paper contributes a Markov Decision Process formulation (MDP) that supports flight envelope protection during in-flight icing. Novel state features based on a state-space abstraction that captures risk related to degradation of aircraft dynamics and controllability as a function of exposure to icing conditions are introduced. 
The rest of the paper is organized as follows. Section II provides a literature review. Section III provides background on Markov Decision Processes. Section IV formulates the FSAM MDP to address in-flight icing conditions. Section V illustrates the application of MDP policies with a case study involving an aircraft experiencing in-flight icing. Finally, Section VI provides conclusions and a brief discussion of future work.

\section{Literature Review}

Several researchers have investigated and developed aircraft ice protection systems. The Smart Icing System (SIS) was developed by Bragg et al. ${ }^{19}$ SIS had capabilities to sense ice accumulation based on its effect on aircraft stability and performance, ${ }^{20}$ adapt flight control laws to accommodate the degraded flight performance ${ }^{21}$ and inform the crew to improve their situational awareness and ability to modify the flight plan as needed. ${ }^{22}$ Gingras et al. ${ }^{23}$ developed the Ice Contamination Envelope Protection (ICEPro) system. ICEPro focused exclusively on estimating degraded airplane performance to inform the flight crew about the degraded flight envelopes via cues presented in the flight displays. Lombaerts et al. ${ }^{24,25}$ developed an icing-related LOC prevention system that was conceptually similar to ICEPro and that predicted envelope violations over a finite horizon.

The primary focus of work cited above was to develop robust identification and control techniques that can adapt to progressive aerodynamic performance changes due to ice accretion and thereby reduce the risk of LOC. All these systems relied on the crew to enter control and flight plan changes that keep the aircraft within a safe operating region and navigate the aircraft to a safe landing or to an atmospheric volume with less or no icing. This work uses the capabilities of the Envelope-Aware FMS as an efficient augmentation to the conventional control authority (i.e. pilot). Consequently, this paper contributes a novel decision making system that assesses risk associated with the current flight condition and selects the appropriate control authority to ensure the aircraft remains within the safe flight envelope. FSAM operates at a higher decision making level enabling flight plan and guidance input changes when flight envelope protection and warnings are not sufficient to mitigate risk along the current flight path.

\section{Markov Decision Process Background}

FSAM is formulated as a Markov Decision Process (MDP) in this paper. A fully observable MDP ${ }^{26}$ is represented as a tuple $(\mathcal{S}, \mathcal{A}, \mathcal{T}, \mathcal{R})$, where $\mathcal{S}$ represents a finite set of system states, $\mathcal{A}$ represents a finite set of actions, $\mathcal{T}$ : $\mathcal{S} \times \mathcal{A} \times \mathcal{S} \rightarrow[0,1]$ captures discrete system dynamics specified as state transition probabilities given selected policy actions, and $\mathcal{R}: \mathcal{S} \times \mathcal{A} \rightarrow \mathbb{R}$ is a reward function over the set of possible state-action pairs. Actions $a_{n} \in \mathcal{A}$ at each decision epoch are chosen such that they maximize the expected cumulative discounted reward function of the form

$$
\mathcal{V}\left(s_{n}\right)=\mathbb{E}\left[\sum_{n=0}^{\infty} \lambda^{n} \mathcal{R}\left(s_{n}, a_{n}\right)\right]
$$

Here, $s_{n}$ is the current state, $a_{n}$ is the action selected at the current state, and $\lambda \in(0,1]$ is a discount factor that weights short-term versus future rewards. The optimal policy $\pi^{*}$ is given by:

$$
\pi^{*}(s)=\underset{a}{\operatorname{argmax}}\{Q(s, a)\}
$$

where $Q(s, a)$ is the utility of a state-action pair and is defined as:

$$
Q(s, a)=\mathcal{R}(s, a)+\sum_{s^{\prime} \in \mathcal{S}} \lambda \mathcal{T}\left(s, a, s^{\prime}\right) \mathcal{V}^{*}\left(s^{\prime}\right)
$$

$\mathcal{V}^{*}(s)$ is the optimal value of state $s$ and can be obtained using algorithms such as value iteration. ${ }^{26,27}$

\section{FSAM MDP Formulation for In-Flight Icing}

\section{IV.A. State formulation}

The ideal MDP state formulation must encode information about aircraft dynamics and controls, aircraft health, pilot and environment characteristics to make override decisions and reduce LOC risk. ${ }^{28}$ Because the full FSAM formulation would be ideally described by a large suite of continuous and discrete variables, abstraction and decomposition are essential to manage complexity. At the top level, the MDP can be decomposed into a sequence of MDP formulations 
for the different phases of flight (i.e. takeoff, climb, cruise, descent and landing). Furthermore, compact abstractions of aircraft performance and flight envelopes pertinent to FSAM override decisions can be constructed to reduce state space complexity for each phase of flight.

This work focuses on icing as the primary hazard, assuming that aircraft health, pilot characteristics and environment features (except icing) do not pose additional risks with respect to flight safety and override. The compact FSAM MDP state representation for icing is defined as the tuple:

$$
s=(\bar{V}, \bar{A}, \bar{\Theta}, \bar{\Phi}, \bar{H}, \bar{T}, \bar{F}, \bar{I}, \bar{S}, \bar{M})
$$

Here $\bar{V}=\left\{\bar{v}_{1}, \ldots, \bar{v}_{6}\right\}$ represents intervals of aircraft airspeed $V$. Each $\bar{v}_{i}$ represents a partition defined over controllable airspeeds in the interval $\left[V_{\max }, V_{\min }\right]$ as illustrated in Eqn (5) and Fig 2. $\bar{v}_{1}$ is a high risk state due to the possibility of aerodynamic stall while $\bar{v}_{6}$ poses high structural damage risk due to high aero-structural loads. $\bar{V}$ encodes information about the proximity to airspeed envelope boundaries.

$$
\begin{aligned}
& \bar{v}_{1}=\left\{V \mid V<V_{\min }\right\} \\
& \bar{v}_{2}=\left\{V \mid 0<V-V_{\min } \leq 0.1 \Delta V\right\} \\
& \bar{v}_{3}=\left\{V \mid 0.1 \Delta V<V-V_{\min } \leq 0.4 \Delta V\right\} \\
& \bar{v}_{4}=\left\{V \mid 0.4 \Delta V<V-V_{\min } \leq 0.8 \Delta V\right\} \\
& \bar{v}_{5}=\left\{V \mid 0.8 \Delta V<V-V_{\min } \leq \Delta V\right\} \\
& \bar{v}_{6}=\left\{V \mid V>V_{\text {max }}\right\}
\end{aligned}
$$

Above, $\Delta V=V_{\max }-V_{\min }$. The graphical representations of the state partitions in Fig 2 are illustrated with instruments found in a typical Cessna C-172 type aircraft. While the values are specific to the $\mathrm{C}-172$ the partition set generalizes to any fixed-wing aircraft type.
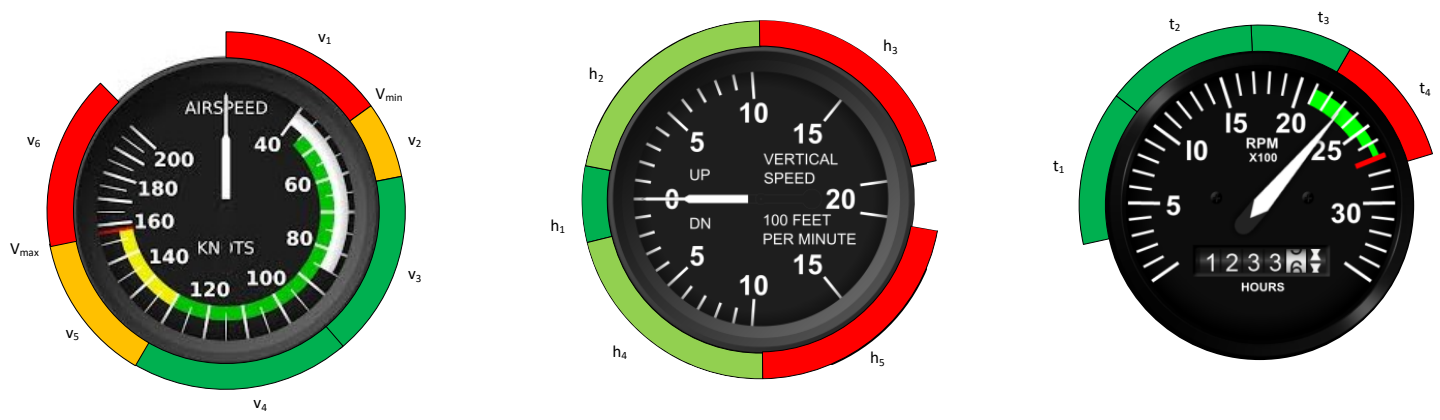

Figure 2. Partitions in Airspeed (left), Vertical speed (center), Thrust (right) ${ }^{29-31}$

$\bar{A}=\left\{\bar{\alpha}_{1}, \bar{\alpha}_{2}, \bar{\alpha}_{3}\right\}$ represents partitions of the adverse aerodynamic envelope boundaries introduced by Wilborn et al. ${ }^{32}$ It encodes information about proximity to a stall condition. Figure 3 illustrates the partitions of the adverse aerodynamic envelope. Note that $\bar{\alpha}_{3}$ represents high risk state where aerodynamic stall is highly likely. Let $X=$ $\left[\alpha_{m}, \beta_{m}\right]$ represent a vector whose components are the normalized angle of attack and sideslip angles. ${ }^{32}$ Adverse aerodynamic envelope abstractions are formally defined as follows:

$$
\begin{aligned}
& \bar{\alpha}_{1}=\left\{X \mid A_{\alpha} X \leq B_{\alpha 1}\right\} \\
& \bar{\alpha}_{2}=\left\{X \mid\left(A_{\alpha} X \leq B_{\alpha 2}\right) \backslash \bar{\alpha}_{1}\right\} \\
& \bar{\alpha}_{3}=\left\{X \mid \mathbb{R}^{2} \backslash\left(A_{\alpha} X \leq B_{\alpha 2}\right)\right\}
\end{aligned}
$$

where $A_{\alpha}, B_{\alpha 1}, B_{\alpha 2}$ are defined as follows:

$$
A_{\alpha}=\left[\begin{array}{cc}
1 & 0 \\
-1 & 0 \\
0 & 1 \\
0 & -1
\end{array}\right], B_{\alpha 1}=\left[\begin{array}{l}
0.80 \\
0.05 \\
0.80 \\
0.80
\end{array}\right], B_{\alpha 2}=\left[\begin{array}{l}
1 \\
0 \\
1 \\
1
\end{array}\right]
$$




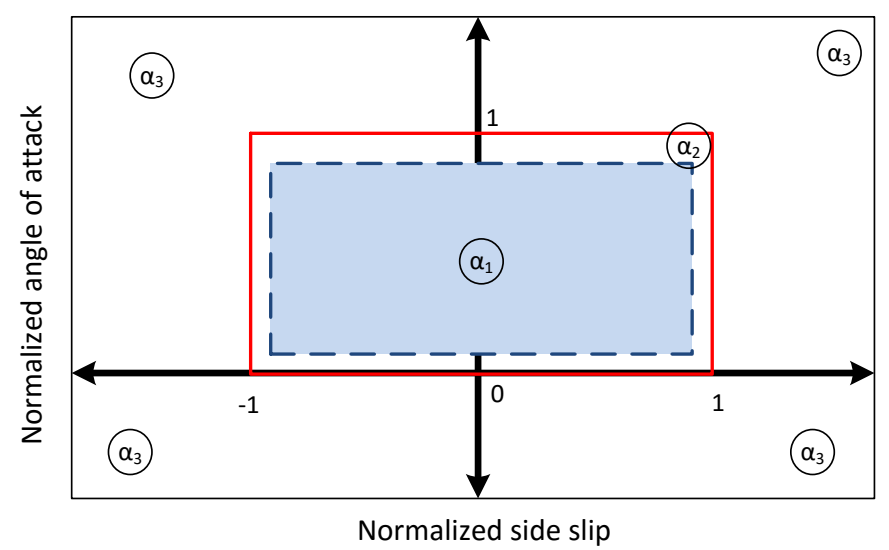

Figure 3. Adverse aerodynamic envelope partitions

$\bar{\Theta} \in\left\{\bar{\theta}_{1}, \bar{\theta}_{2}, \bar{\theta}_{3}, \bar{\theta}_{4}, \bar{\theta}_{5}, \bar{\theta}_{6}\right\}$ is a compact representation of aircraft pitch $\theta$, pitch rate $q$ and elevator control $\delta_{e}$. Specifically, $\bar{\Theta}$ is a discretization of the dynamic pitch control envelope introduced by Wilborn et al. ${ }^{32}$ The dynamic pitch $\theta^{\prime}$ is defined as $\theta+q$. $\Theta$ is illustrated in Fig 4. Let $X=\left[\theta^{\prime}, \delta_{e}\right]$ represent a vector whose components are the dynamic pitch attitude and elevator deflection. ${ }^{32}$ Dynamic pitch control envelope abstractions are formally defined as follows:

$$
\begin{aligned}
& \bar{\theta}_{1}=\left\{X \mid A_{\theta} X \leq B_{\theta 1}\right\} \\
& \bar{\theta}_{2}=\left\{X \mid\left(A_{\theta} X \leq B_{\theta 2}\right) \backslash \bar{\theta}_{1}\right\} \\
& \bar{\theta}_{3}=\left\{X \mid Q_{1} \backslash \bar{\theta}_{2}\right\} \\
& \bar{\theta}_{4}=\left\{X \mid Q_{2} \backslash \bar{\theta}_{2}\right\} \\
& \bar{\theta}_{5}=\left\{X \mid Q_{3} \backslash \bar{\theta}_{2}\right\} \\
& \bar{\theta}_{6}=\left\{X \mid Q_{4} \backslash \bar{\theta}_{2}\right\}
\end{aligned}
$$

where $Q_{i}, i=1,2,3,4$ denote the first, second, third and fourth quadrants in $\mathbb{R}^{2} . A_{\theta}, B_{\theta 1}, B_{\theta 2}$ are defined as follows:

$$
A_{\theta}=\left[\begin{array}{cc}
1 & 0 \\
-1 & 0 \\
0 & 1 \\
0 & -1 \\
-m_{1} & 1 \\
m_{2} & -1
\end{array}\right], B_{\theta 1}=\left[\begin{array}{c}
\delta_{e_{\max }} \\
\theta_{\max } \\
\delta_{e_{\min }} \\
\theta_{\min } \\
\theta_{\max } \\
\theta_{\min }
\end{array}\right], B_{\theta 2}=0.8 B_{\theta 1}
$$

where $m_{1}, m_{2}$ are the slopes of the Figure 4 boundary lines in $Q_{2}, Q_{4}$ respectively. $\delta_{e_{\min }}, \delta_{e_{\max }}$ represent elevator saturation limits while $\theta_{\min }, \theta_{\max }$ represent safe pitch attitude limits.

$\bar{\Phi} \in\left\{\bar{\phi}_{1}, \ldots, \bar{\phi}_{6}\right\}$ is a compact representation of aircraft roll $\phi$, roll rate $p$ and aileron control $\delta_{a}$. Dynamic roll $\phi^{\prime}$ is defined as $\phi+p . \bar{\Phi}$ is a discretization of the dynamic roll control envelope as specified in Wilborn et al. ${ }^{32} \bar{\phi}_{1}, \bar{\phi}_{2}$ represent safe operating envelope regions. The dynamic roll control envelope partitions are defined as follows:

$$
\begin{aligned}
& \bar{\phi}_{1}=\left\{X \mid A_{\phi} X \leq B_{\phi 1}\right\} \\
& \bar{\phi}_{2}=\left\{X \mid\left(A_{\phi} X \leq B_{\phi 2}\right) \backslash \bar{\phi}_{1}\right\} \\
& \bar{\phi}_{3}=\left\{X \mid Q_{1} \backslash \bar{\phi}_{2}\right\} \\
& \bar{\phi}_{4}=\left\{X \mid Q_{2} \backslash \bar{\phi}_{2}\right\} \\
& \bar{\phi}_{5}=\left\{X \mid Q_{3} \backslash \bar{\phi}_{2}\right\} \\
& \bar{\phi}_{6}=\left\{X \mid Q_{4} \backslash \bar{\phi}_{2}\right\}
\end{aligned}
$$




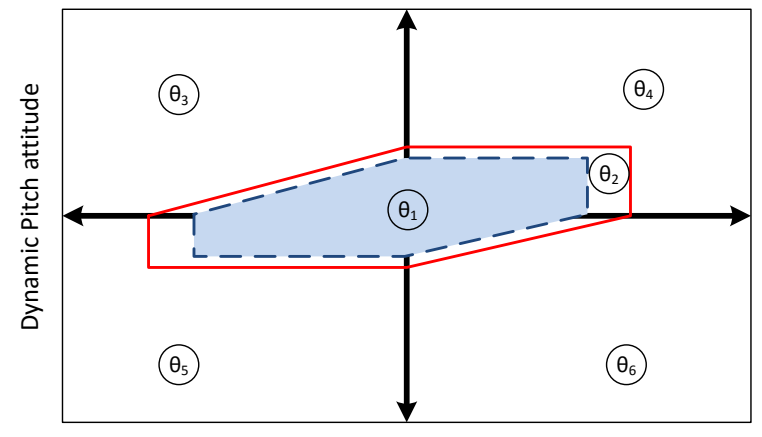

Percentage Pitch Control

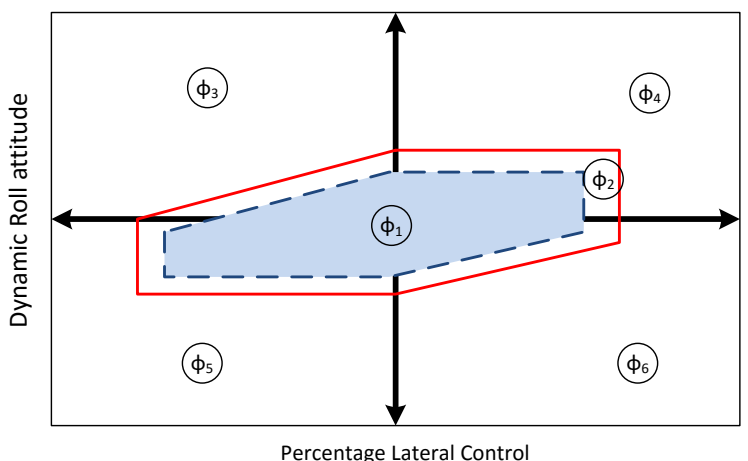

Percentage Lateral Control

Figure 4. Dynamic pitch and roll control envelope partitions

$A_{\phi}, B_{\phi 1}, B_{\phi 2}$ are defined as follows:

$$
A_{\phi}=\left[\begin{array}{cc}
1 & 0 \\
-1 & 0 \\
0 & 1 \\
0 & -1 \\
-m_{1} & 1 \\
m_{2} & -1
\end{array}\right], B_{\phi 1}=\left[\begin{array}{l}
\delta_{a_{\max }} \\
\phi_{\max } \\
\delta_{a_{\min }} \\
\phi_{\min } \\
\phi_{\max } \\
\phi_{\min }
\end{array}\right], B_{\phi 2}=0.8 B_{\phi 1}
$$

where $m_{1}, m_{2}$ are the slopes of the Figure 4 boundary lines in $Q_{2}, Q_{4}$ respectively. $\delta_{a_{\min }}, \delta_{a_{\max }}$ represent aileron saturation limits while $\phi_{\min }, \phi_{\max }$ represent safe roll attitude limits.

$\bar{H}=\left\{\bar{h}_{1}, \ldots, \bar{h}_{5}\right\}$ characterizes partitions of the aircraft's vertical speed as shown in Fig 2. Let $\dot{h}_{\max }, \dot{h}_{\min }$ denote the maximum and minimum climb rates for safe operation and let $\dot{h}_{0}>0$ denote an appropriate small value of climb rate close to zero. Partition intervals are defined as follows:

$$
\begin{aligned}
& \bar{h}_{1}=\left\{\dot{h}|| \dot{h} \mid<\dot{h}_{0} \mathrm{ft} / \mathrm{min}\right\} \\
& \bar{h}_{2}=\left\{\dot{h} \mid \dot{h}_{0} \mathrm{ft} / \min \leq \dot{h}<\dot{h}_{\max } \mathrm{ft} / \mathrm{min}\right\} \\
& \bar{h}_{3}=\left\{\dot{h} \mid \dot{h} \geq \dot{h}_{\max } \mathrm{ft} / \mathrm{min}\right\} \\
& \bar{h}_{4}=\left\{\dot{h} \mid-\dot{h}_{0} \mathrm{ft} / \min \geq \dot{h}>\dot{h}_{\min } \mathrm{ft} / \mathrm{min}\right\} \\
& \bar{h}_{5}=\left\{\dot{h} \mid \dot{h} \leq \dot{h}_{\min } \mathrm{ft} / \mathrm{min}\right\}
\end{aligned}
$$

$\bar{T} \in\left\{\bar{t}_{1}, \bar{t}_{2}, \bar{t}_{3}, \bar{t}_{4}\right\}$ denote partitions of the thrust control input space as shown in Fig 2. $T_{\max }$ denotes the maximum thrust output and the partitions are defined as follows:

$$
\begin{aligned}
& \bar{t}_{1}=\left\{T \mid 0 \leq T \leq 0.45 T_{\text {max }}\right\} \\
& \bar{t}_{2}=\left\{T \mid 0.45 T_{\text {max }}<T \leq 0.75 T_{\max }\right\} \\
& \bar{t}_{3}=\left\{T \mid 0.75 T_{\text {max }}<T \leq 0.98 T_{\text {max }}\right\} \\
& \bar{t}_{4}=\left\{T \mid 0.98 T_{\text {max }}<T \leq T_{\text {max }}\right\}
\end{aligned}
$$

$\bar{F}$ represents current flight plan information with triple $\left(F_{c}, F_{t}, F_{s}\right)$ that characterizes climb, turn and airspeed.

$$
\begin{aligned}
& F_{c} \in\{\text { level,climb,descent }\} \\
& F_{t} \in\{\text { straight,turn }\} \\
& F_{s} \in\{\text { slow,med,fast }\}
\end{aligned}
$$

Values 'level', 'climb' and 'descent' are defined as flight conditions with zero, positive and negative climb rates, respectively. 'Straight' and 'turn' are defined as flight conditions with zero and non-zero turn rates, respectively. 'Slow' 
Table 1. Flight plan state composition

\begin{tabular}{|c|c|c|c|c|c|}
\hline $\bar{f}_{1}$ & (level,straight,slow) & $\bar{f}_{2}$ & (level,straight,med) & $\bar{f}_{3}$ & (level,straight,fast) \\
\hline $\bar{f}_{4}$ & (level,turn,slow) & $\bar{f}_{5}$ & (level,turn,med) & $\bar{f}_{6}$ & (level,turn,fast) \\
\hline $\bar{f}_{7}$ & (climb,straight,slow) & $\bar{f}_{8}$ & (climb,straight,med) & $\bar{f}_{9}$ & (climb,straight,fast) \\
\hline $\bar{f}_{10}$ & (climb,turn,slow) & $\bar{f}_{11}$ & (climb,turn,med) & $\bar{f}_{12}$ & (climb,turn,fast) \\
\hline $\bar{f}_{13}$ & (descent,straight,slow) & $\bar{f}_{14}$ & (descent,straight,med) & $\bar{f}_{15}$ & (descent,straight,fast) \\
\hline $\bar{f}_{16}$ & (descent,turn,slow) & $\bar{f}_{17}$ & (descent,turn,med) & $\bar{f}_{18}$ & (descent,turn,fast) \\
\hline
\end{tabular}

Table 2. Icing intensity state abstraction

\begin{tabular}{|c|c|}
\hline $\bar{i}_{0}$ & $\left(0<t_{\mathrm{pte}}<t_{\text {critical }}, 0\right)$ \\
\hline $\bar{i}_{1}$ & $\left(t_{\mathrm{pte}} \geq t_{\text {critical }}, 0\right)$ \\
\hline $\bar{i}_{2}$ & $\left(0<t_{\mathrm{pte}}<t_{\text {critical }}, 1\right)$ \\
\hline $\bar{i}_{3}$ & $\left(t_{\mathrm{pte}} \geq t_{\text {critical }}, 1\right)$ \\
\hline $\bar{i}_{4}$ & $\left(t_{\mathrm{pte}}=0,0\right)$ \\
\hline
\end{tabular}

is defined as the set of flight conditions where $\bar{V} \in\left\{\bar{v}_{1}, \bar{v}_{2}\right\}$, 'med' includes flight states with $\bar{V} \in\left\{\bar{v}_{3}, \bar{v}_{4}\right\}$ and 'fast' states have $\bar{V} \in\left\{\bar{v}_{5}, \bar{v}_{6}\right\}$. Thus, $\bar{F}$ is abstracted into $\left\{\bar{f}_{1}, \ldots, \bar{f}_{18}\right\}$ as shown in Table 1$)$.

$\bar{I}$ encapsulates information about predicted exposure to icing conditions based on a given flight plan and expected atmospheric (icing, wind) conditions. Let $t_{\text {pte }}$ be defined as the predicted time of exposure to icing. The critical exposure time $t_{\text {critical }}$ is defined as the duration beyond which further exposure to icing condition is most likely to result in stall conditions. Note that the critical exposure time depends on several factors such as icing severity, maximum thrust available, commanded airspeed during icing conditions, usage of deicing fluids prior to takeoff, and capacity of the anti-icing system. ${ }^{33} \bar{I}$ is defined as the tuple $\left(n_{\text {pte }}, n_{\text {ice }}\right)$ where $n_{\text {pte }} \in\left\{t_{\text {pte }}=0,0<t_{\text {pte }}<t_{\text {critical }}, t_{\text {pte }}>t_{\text {critical }}\right\}$ denotes partitions in the predicted time of exposure and $n_{\text {ice }} \in\{0,1\}$ where 0 denotes flight outside icing clouds and 1 denotes that the aircraft is flying in icing conditions. $\bar{I}$ is compactly represented as $\left\{\bar{i}_{0}, \bar{i}_{1}, \bar{i}_{2}, \bar{i}_{3}, \bar{i}_{4}\right\}$ as shown in Table 2. Figure 5 graphically illustrates these states.
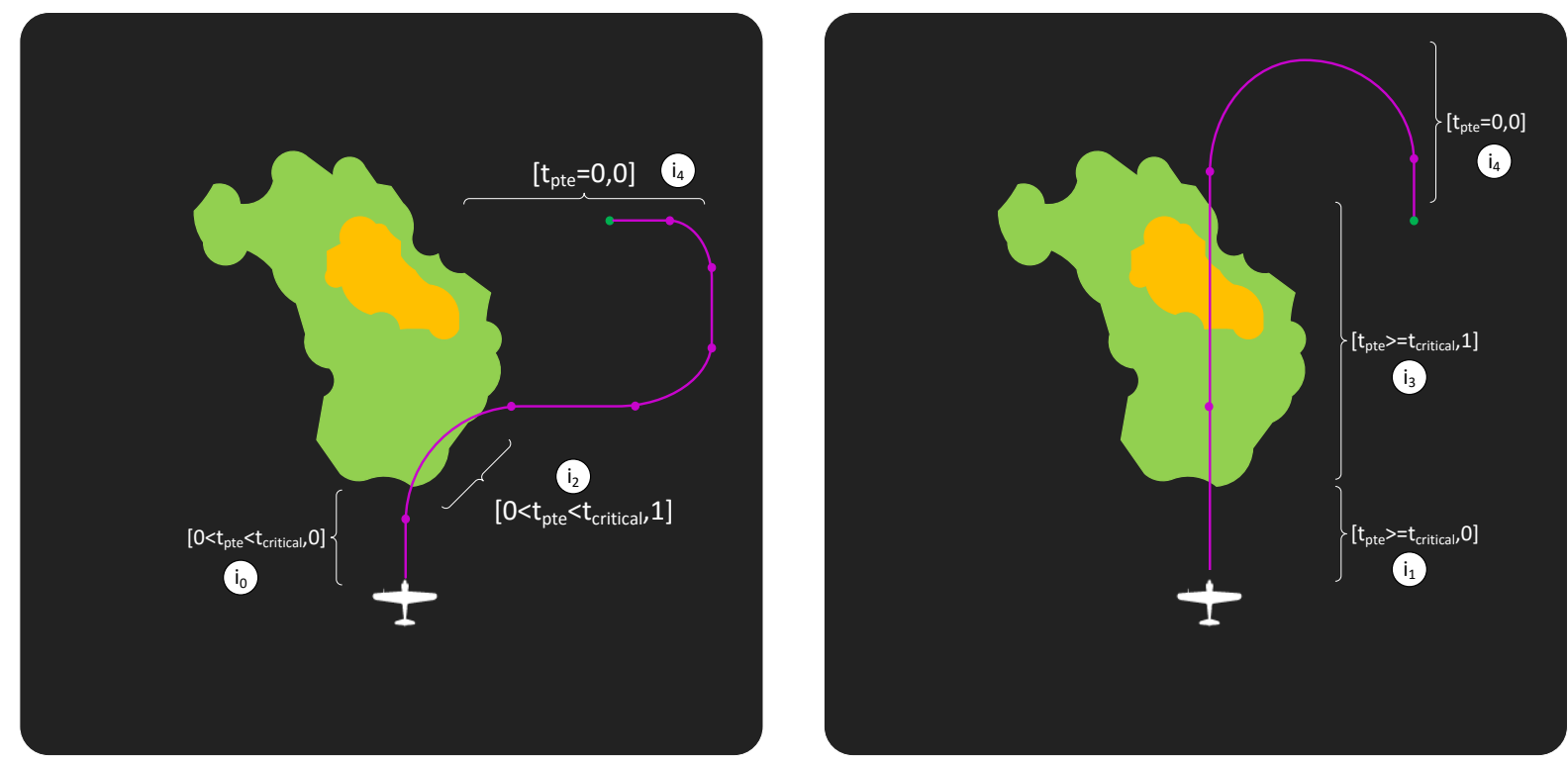

Figure 5. Abstraction for icing intensity based on available flight plan

$\bar{M} \in\{P, E A\}$ represents the current control mode. Here $P$ denotes that the pilot is in control while $E A$ indicates envelope-aware control. $\bar{S} \in\left\{P_{s}, E A_{s}\right\}$ represents a mode select switch with which the pilot can request pilot control authority be maintained or restored $\left(P_{s}\right)$ or can manually engage or maintain Envelope-Aware control $\left(E A_{s}\right)$. 
Note that the airspeed, angle of attack, pitch and roll partitions described above are parametrized in terms of envelope boundaries. Consequently, as these parameters are updated by envelope estimation, these state representations capture the evolving risks due to icing.

\section{IV.B. Action Set}

FSAM is a high-level watchdog that passively monitors for LOC risk and overrides only when necessary to avoid LOC. The FSAM MDP defines a policy over two actions: NOOP (No Operation) and TOGL (Toggle). Any time FSAM selects NOOP current control mode $M$ remains engaged. If the current control mode $M$ indicates nominal pilot/autopilot authority and FSAM selects the TOGL action, FSAM activates the envelope-aware controller. If the current control mode $M$ is the envelope-aware controller and FSAM selects the TOGL action, authority is returned to the nominal pilot/autopilot system. The FSAM actions defined here are consistent with our previous work. ${ }^{28}$ The pilot could also request control back or activate the envelope-aware controller via the mode select switch $\bar{S}$. The value of $\bar{S}$ biases the FSAM policy towards satisfying the pilot's request. Section IV.D illustrates how $\bar{S}$ influences MDP reward.

\section{IV.C. Transition Probabilities}

State transition dynamics can be modeled as a Markov chain with consideration for each FSAM MDP policy control authority decision (NOOP or TOGL). Because transition dynamics fundamentally evolve as a function of current control mode $M$ one transition probability matrix is defined for each control mode $M$. Switching between the two probability tables for $M=P$ and $M=E A$ then occurs for each state in which the MDP selects the TOGL action.

Let $\overline{\mathcal{T}}_{M}, M \in\{P, E A\}$ define the two Markov chain transition matrices. Given the state abstractions in Section IV, the underlying continuous time process remains in a state $s_{k} \in \mathcal{S}$ for some duration called the sojourn time $\sigma\left(s_{k}\right) . \sigma$ is modeled as an exponential distribution with parameter $\beta\left(s_{k}\right)$. Estimated values of $\beta\left(s_{k}\right)$ and $\overline{\mathcal{T}}_{M}\left(s_{j} \mid s_{i}\right), s_{i}, s_{j} \in \mathcal{S}$ can be computed from flight/simulation data.

$$
\begin{gathered}
\beta\left(s_{k}\right)=\mathbb{E}\left[\frac{1}{\sigma\left(s_{k}\right)}\right] \\
\overline{\mathcal{T}}_{M}\left(s_{j} \mid s_{i}\right)=\frac{N\left(s_{i}, s_{j}\right)}{\sum_{n} N\left(s_{i}, s_{n}\right)}, \text { where } n=1, \ldots,|\mathcal{S}|
\end{gathered}
$$

Here $N\left(s_{i}, s_{j}\right)$ represents the total number of transitions from state $s_{i}$ to $s_{j}$. The above Markov chain has statedependent sojourn times. A discrete-time Markov chain can be transformed into an equivalent Markov chain $\mathcal{T}_{M}$ whose sojourn time distributions are identical for all states through a uniformization process ${ }^{26}$ in which the discrete time Markov chain $\mathcal{T}_{M}$ is described by:

$$
\mathcal{T}_{M}\left(s_{j} \mid s_{i}\right)=\left\{\begin{array}{cc}
1-\frac{1}{c}\left(1-\overline{\mathcal{T}}_{M}\left(s_{i} \mid s_{i}\right)\right) \beta\left(s_{i}\right) & \text { if } s_{i}=s_{j} \\
\frac{1}{c}\left(\overline{\mathcal{T}}_{M}\left(s_{j} \mid s_{i}\right) \beta\left(s_{i}\right)\right) & \text { otherwise }
\end{array}\right.
$$

where $c=\max _{s_{i} \in \mathcal{S}}\left\{1-\overline{\mathcal{T}}_{M}\left(s_{j} \mid s_{i}\right) \beta\left(s_{i}\right)\right\}$ is the sojourn time distribution parameter for the new Markov chain.

An alternate method of evaluating $\mathcal{T}_{M}$ is to consider each state as the composition of its individual state features. Consequently, the required probability distribution can be expressed in terms of the individual state features as follows:

$$
\overline{\mathcal{T}}_{M}\left(s_{j} \mid s_{i}\right)=\overline{\mathcal{P}}\left(\bar{V}_{j}, \bar{A}_{j}, \bar{\Theta}_{j}, \bar{\Phi}_{j}, \bar{H}_{j}, \bar{T}_{j}, \bar{F}_{j}, \bar{I}_{j}, \bar{S}_{j} \mid \bar{V}_{i}, \bar{A}_{i}, \bar{\Theta}_{i}, \bar{\Phi}_{i}, \bar{H}_{i}, \bar{T}_{i}, \bar{F}_{i}, \bar{I}_{i}, \bar{S}_{i}, M\right)
$$


Using the product rule ${ }^{\mathrm{a}}$ Eqn (9) can be written as follows:

$$
\begin{aligned}
\overline{\mathcal{T}}_{M}\left(s_{j} \mid s_{i}\right) & =\overline{\mathcal{P}}_{1}\left(\bar{V}_{j} \mid \bar{A}_{j}, \bar{\Theta}_{j}, \bar{\Phi}_{j}, \bar{H}_{j}, \bar{T}_{j}, \bar{F}_{j}, \bar{I}_{j}, \bar{S}_{j}, \bar{V}_{i}, \bar{A}_{i}, \bar{\Theta}_{i}, \bar{\Phi}_{i}, \bar{H}_{i}, \bar{T}_{i}, \bar{F}_{i}, \bar{I}_{i}, \bar{S}_{i}, M\right) \\
& \times \overline{\mathcal{P}}_{2}\left(\bar{A}_{j} \mid \bar{\Theta}_{j}, \bar{\Phi}_{j}, \bar{H}_{j}, \bar{T}_{j}, \bar{F}_{j}, \bar{I}_{j}, \bar{S}_{j}, \bar{V}_{i}, \bar{A}_{i}, \bar{\Theta}_{i}, \bar{\Phi}_{i}, \bar{H}_{i}, \bar{T}_{i}, \bar{F}_{i}, \bar{I}_{i}, \bar{S}_{i}, M\right) \\
& \times \overline{\mathcal{P}}_{3}\left(\bar{\Theta}_{j} \mid \bar{\Phi}_{j}, \bar{H}_{j}, \bar{T}_{j}, \bar{F}_{j}, \bar{I}_{j}, \bar{S}_{j}, \bar{V}_{i}, \bar{A}_{i}, \bar{\Theta}_{i}, \bar{\Phi}_{i}, \bar{H}_{i}, \bar{T}_{i}, \bar{F}_{i}, \bar{I}_{i}, \bar{S}_{i}, M\right) \\
& \times \overline{\mathcal{P}}_{4}\left(\bar{\Phi}_{j} \mid \bar{H}_{j}, \bar{T}_{j}, \bar{F}_{j}, \bar{I}_{j}, \bar{S}_{j}, \bar{V}_{i}, \bar{A}_{i}, \bar{\Theta}_{i}, \bar{\Phi}_{i}, \bar{H}_{i}, \bar{T}_{i}, \bar{F}_{i}, \bar{I}_{i}, \bar{S}_{i}, M\right) \\
& \times \overline{\mathcal{P}}_{5}\left(\bar{H}_{j} \mid \bar{T}_{j}, \bar{F}_{j}, \bar{I}_{j}, \bar{S}_{j}, \bar{V}_{i}, \bar{A}_{i}, \bar{\Theta}_{i}, \bar{\Phi}_{i}, \bar{H}_{i}, \bar{T}_{i}, \bar{F}_{i}, \bar{I}_{i}, \bar{S}_{i}, M\right) \\
& \times \overline{\mathcal{P}}_{6}\left(\bar{T}_{j} \mid \bar{F}_{j}, \bar{I}_{j}, \bar{S}_{j}, \bar{V}_{i}, \bar{A}_{i}, \bar{\Theta}_{i}, \bar{\Phi}_{i}, \bar{H}_{i}, \bar{T}_{i}, \bar{F}_{i}, \bar{I}_{i}, \bar{S}_{i}, M\right) \\
& \times \overline{\mathcal{P}}_{7}\left(\bar{F}_{j} \mid \bar{I}_{j}, \bar{S}_{j}, \bar{V}_{i}, \bar{A}_{i}, \bar{\Theta}_{i}, \bar{\Phi}_{i}, \bar{H}_{i}, \bar{T}_{i}, \bar{F}_{i}, \bar{I}_{i}, \bar{S}_{i}, M\right) \\
& \times \overline{\mathcal{P}}_{8}\left(\bar{I}_{j} \mid \bar{S}_{j}, \bar{V}_{i}, \bar{A}_{i}, \bar{\Theta}_{i}, \bar{\Phi}_{i}, \bar{H}_{i}, \bar{T}_{i}, \bar{F}_{i}, \bar{I}_{i}, \bar{S}_{i}, M\right) \\
& \times \overline{\mathcal{P}}_{9}\left(\bar{S}_{j} \mid \bar{V}_{i}, \bar{A}_{i}, \bar{\Theta}_{i}, \bar{\Phi}_{i}, \bar{H}_{i}, \bar{T}_{i}, \bar{F}_{i}, \bar{I}_{i}, \bar{S}_{i}, M\right)
\end{aligned}
$$

Eqn (10) can be further simplified by by examining state feature conditional independence. This work assumes the sojourn time between states is small. In this case state features at the next time step $j$ can be assumed to be conditionally independent of the state features at the current time step $i{ }^{\mathrm{b}}$ This simplified expression for transition probability is:

$$
\begin{aligned}
\mathcal{T}_{M}\left(s_{j} \mid s_{i}\right) & =\mathcal{P}_{1}\left(\bar{V}_{j} \mid \bar{V}_{i}, \bar{A}_{i}, \bar{\Theta}_{i}, \bar{\Phi}_{i}, \bar{H}_{i}, \bar{T}_{i}, \bar{F}_{i}, \bar{I}_{i}, M\right) \times \mathcal{P}_{2}\left(\bar{A}_{j} \mid \bar{V}_{i}, \bar{A}_{i}, \bar{\Theta}_{i}, \bar{\Phi}_{i}, \bar{H}_{i}, \bar{T}_{i}, \bar{F}_{i}, \bar{I}_{i}, M\right) \\
& \times \mathcal{P}_{3}\left(\bar{\Theta}_{j} \mid \bar{V}_{i}, \bar{A}_{i}, \bar{\Theta}_{i}, \bar{\Phi}_{i}, \bar{H}_{i}, \bar{T}_{i}, \bar{F}_{i}, \bar{I}_{i}, M\right) \times \mathcal{P}_{4}\left(\bar{\Phi}_{j} \mid \bar{V}_{i}, \bar{A}_{i}, \bar{\Theta}_{i}, \bar{\Phi}_{i}, \bar{H}_{i}, \bar{T}_{i}, \bar{F}_{i}, \bar{I}_{i}, M\right) \\
& \times \mathcal{P}_{5}\left(\bar{H}_{j} \mid \bar{V}_{i}, \bar{A}_{i}, \bar{\Theta}_{i}, \bar{\Phi}_{i}, \bar{H}_{i}, \bar{T}_{i}, \bar{F}_{i}, \bar{I}_{i}, M\right) \times \mathcal{P}_{6}\left(\bar{T}_{j} \mid \bar{V}_{i}, \bar{A}_{i}, \bar{\Theta}_{i}, \bar{\Phi}_{i}, \bar{H}_{i}, \bar{T}_{i}, \bar{F}_{i}, \bar{I}_{i}, M\right) \\
& \times \mathcal{P}_{7}\left(\bar{F}_{j} \mid \bar{V}_{i}, \bar{A}_{i}, \bar{\Theta}_{i}, \bar{\Phi}_{i}, \bar{H}_{i}, \bar{T}_{i}, \bar{F}_{i}, M\right) \times \mathcal{P}_{8}\left(\bar{I}_{j} \mid \bar{I}_{i}, M\right) \times \mathcal{P}_{9}\left(\bar{S}_{j} \mid \bar{S}_{i}, M\right)
\end{aligned}
$$

Note that $\mathcal{P}_{i=1, \ldots, 9}$ are the uniformized distributions where uniformization is carried out as described in Eqn (8) with parameter $c$ chosen as $\max _{s_{i} \in \mathcal{S}}\left\{1-\overline{\mathcal{T}}\left(s_{j} \mid s_{i}\right) \beta\left(s_{i}\right)\right\}$. The factored representation of $\mathcal{T}_{M}$ provides additional flexibility since this facilitates the incorporation of data from several sources. Each distribution can be estimated via one or more methods such as Monte Carlo sampling of physics-based models, flight data mining, and human subject experiments.

Let $\mathcal{T}_{N O O P}$ and $\mathcal{T}_{T O G L}$ denote the transition probability matrices for $a=N O O P$ and $a=T O G L$, respectively. The state features in Eqn (4) are permuted such that $\mathcal{T}_{N O O P}$ and $\mathcal{T}_{T O G L}$ are block diagonal matrices of the form:

$$
\mathcal{T}_{\text {NOOP }}=\left[\begin{array}{cc}
\mathcal{T}_{M=P} & 0 \\
0 & \mathcal{T}_{M=E A}
\end{array}\right], \mathcal{T}_{T O G L}=\left[\begin{array}{cc}
0 & \mathcal{T}_{M=E A} \\
\mathcal{T}_{M=P} & 0
\end{array}\right]
$$

where $\mathcal{T}_{M=P}, \mathcal{T}_{M=E A}$ are the Markov chains for pilot and envelope-aware control modes, respectively.

\section{IV.D. Reward formulation}

FSAM's goal is to ensure that the aircraft avoids states with high LOC risk while minimizing authority shifts away from pilot-designated mode $\bar{S}$. Consequently, an additive reward $\mathcal{R}(s, a)=\sum_{i} w_{i} \mathcal{R}_{i}$ is defined where the $\mathcal{R}_{i}$ 's penalize unsafe states and inconsistent authority switches while the $w_{i}$ are weighting parameters.

$$
\begin{gathered}
\mathcal{R}_{1}=\left\{\begin{array}{l}
-1 \text { if } \bar{V} \in\left\{\bar{v}_{1}, \bar{v}_{6}\right\} \\
0 \text { otherwise }
\end{array} \quad \mathcal{R}_{2}=\left\{\begin{array}{l}
-1 \text { if } \bar{A} \in\left\{\bar{\alpha}_{3}\right\} \\
0 \text { otherwise }
\end{array}\right.\right. \\
\mathcal{R}_{3}=\left\{\begin{array}{l}
-1 \text { if } \bar{\Theta} \in\left\{\bar{\theta}_{3}, \bar{\theta}_{4}, \bar{\theta}_{5}, \bar{\theta}_{6}\right\} \\
0 \text { otherwise }
\end{array} \quad \mathcal{R}_{4} \begin{array}{l}
-1 \text { if } \bar{\Phi} \in\left\{\bar{\phi}_{3}, \bar{\phi}_{4}, \bar{\phi}_{5}, \bar{\phi}_{6}\right\} \\
0 \text { otherwise }
\end{array}\right. \\
\mathcal{R}_{5}=\left\{\begin{array}{l}
-1 \text { if } \bar{H} \in\left\{\bar{h}_{5}\right\} \\
0 \text { otherwise }
\end{array}\right.
\end{gathered}
$$

a Product rule: $\mathcal{P}(x, y \mid z)=\mathcal{P}(x \mid y, z) \mathcal{P}(y \mid z)$, Conditional independence: $\mathcal{P}(x \mid y)=\mathcal{P}(x)$

${ }^{\mathrm{b}}$ This small sojourn time assumption may not always be valid, but further analysis is beyond the scope of this paper. 


$$
\mathcal{R}_{6}=\left\{\begin{array}{l}
-o_{1} \text { if } M=P \wedge \bar{S}=P_{s} \wedge a=T O G L \\
-o_{2} \text { if } M=P \wedge \bar{S}=E A_{s} \wedge a=N O O P \\
-o_{3} \text { if } M=E A \wedge \bar{S}=P_{s} \wedge a=N O O P \\
-o_{4} \text { if } M=E A \wedge \bar{S}=E A_{s} \wedge a=T O G L \\
0 \text { otherwise }
\end{array}\right.
$$

Above, $o_{i=1, \ldots, 4} \in[0,1]$. Note that setting $\left(o_{1}, o_{2}, o_{3}, o_{4}\right)=(1,0,1,0)$ only discourages $E A$ mode when the crew selects $P_{s}$. Persistence in envelope-aware control mode might be primarily penalized to encourage transfer of authority to the crew once any high-risk condition prompting FSAM TOGL to $M=E A$ is mitigated. $\left(o_{1}, o_{2}, o_{3}, o_{4}\right)=(1,1,1,1)$ encourages the policy to satisfy the crew's mode select request. For the case study discussed in this work, the following parameters are chosen: $w_{1}=100, w_{2}=100, w_{3}=50, w_{4}=50, w_{5}=100, w_{6}=10$ and $o_{1}=o_{2}=o_{3}=o_{4}=1$.

\section{IV.E. FSAM MDP policy}

The optimal value $\mathcal{V}^{*}\left(s_{i}\right)$ for each state $s_{i} \in \mathcal{S}$ is obtained using Value Iteration. ${ }^{26}$ In this work, $\mathcal{V}^{*}$ is computed offline. The optimal action at each state $\pi^{*}\left(s_{i}\right)$ is then chosen online according to the following rule:

$$
\pi^{*}\left(s_{i}\right)=\underset{a}{\operatorname{argmax}}\left\{Q\left(s_{i}, a=N O O P\right), Q\left(s_{i}, a=T O G L\right)\right\}
$$

where

$$
Q\left(s_{i}, a\right)=\mathcal{R}\left(s_{i}, a\right)+\lambda \sum_{s_{k} \in \mathcal{S}} \mathcal{T}_{a}\left(s_{k} \mid s_{i}\right) \mathcal{V}^{*}\left(s_{k}\right)
$$

For convenience, Eqn (18) is implemented as follows:

$$
Q\left(s_{i}, a\right)=\mathcal{R}\left(s_{i}, a\right)+\lambda \sum_{s_{k}} \mathcal{T}_{M}\left(s_{k} \mid s_{j}\right) \mathcal{V}^{*}\left(s_{k}\right)
$$

Eqn (19) is equivalent to Eqn (18) because of the block diagonal structure of $\mathcal{T}_{a}$ from Eqn (12). In Eqn (19), $M \in$ $\{P, E A\}$ in $\mathcal{T}_{M}$ is also specified in state $s_{j} . s_{j}=s_{i}$ if the policy prescribes $a=N O O P$ in state $s_{i}$ while the $M$ value is toggled in $s_{j}$ relative to $s_{i}$ if $a=T O G L$. Note that if switching control authorities with TOGL results in a new flight plan with a different predicted exposure time to icing, then $s_{j}$ also allows for instantaneous changes in flight plan and icing intensity feature values relative to $s_{i}{ }^{\mathrm{c}}$

\section{In-flight Icing Case Study}

Consider an aircraft on approach to Buffalo Niagara International Airport ${ }^{\mathrm{d}}$ (KBUF) Runway 23 as shown in Fig 6. The flight plan progresses nominally as follows. From Initial Approach Fix (IAF) SUSKE to BUFST the aircraft maintains steady level flight at a medium speed $\left(\bar{F}=\bar{f}_{2}\right)$. From BUFST onwards, the aircraft starts a straight descent at medium speed $\left(\bar{F}=\bar{f}_{14}\right)$. At ZADUM the aircraft starts a descending right turn $\left(\bar{F}=\bar{f}_{17}\right)$ toward Final Approach Fix (FAF) BIILS and then continues with a straight descent while decelerating $\left(\bar{F}=\bar{f}_{13}\right)$ to a nominal touchdown speed. The construction of state transition probabilities is explained in the below Appendix. Note that to succinctly describe and compute transition probability distributions, this work assumes that the angle of attack, side-slip and dynamic pitch attitude always stay within the safe operating envelope (i.e. $\bar{A}=\bar{\alpha}_{1}, \bar{\Theta}=\bar{\theta}_{1}$ ). The mode select switch is always assumed set to request pilot authority (i.e. $\bar{S}=P_{s}$ ). With the state transition probabilities described in the Appendix and the reward formulation described in Section IV.D, the optimal policy for the MDP is obtained using value iteration. The optimal values for select states relevant to this case study are listed in the Appendix (Table 11).

\section{V.A. Flight without icing conditions}

Consider the case where $\bar{I}=\bar{i}_{4}$. i.e. predicted time of exposure $t_{p t e}=0$ and the aircraft is free from icing conditions $n_{i c e}=0$. Suppose the aircraft is following the flight segment between SUSKE and BUFST with straight and level

${ }^{\mathrm{c}}$ The MDP formulation in this work assumes that the $T O G L$ action only influences the control mode $M$ (see Eqn 12). Consequently, when there is a change in flight plan due to a TOGL action, Eqn (19) results in $Q$ values that are different from the ones computed using value iteration. However, since a new flight plan is only available when the current flight plan is unsafe, the action selected always favors the lower risk state.

${ }^{\mathrm{d}}$ This case study is motivated by the crash of Colgan Air Flight $3407 .{ }^{34}$ 


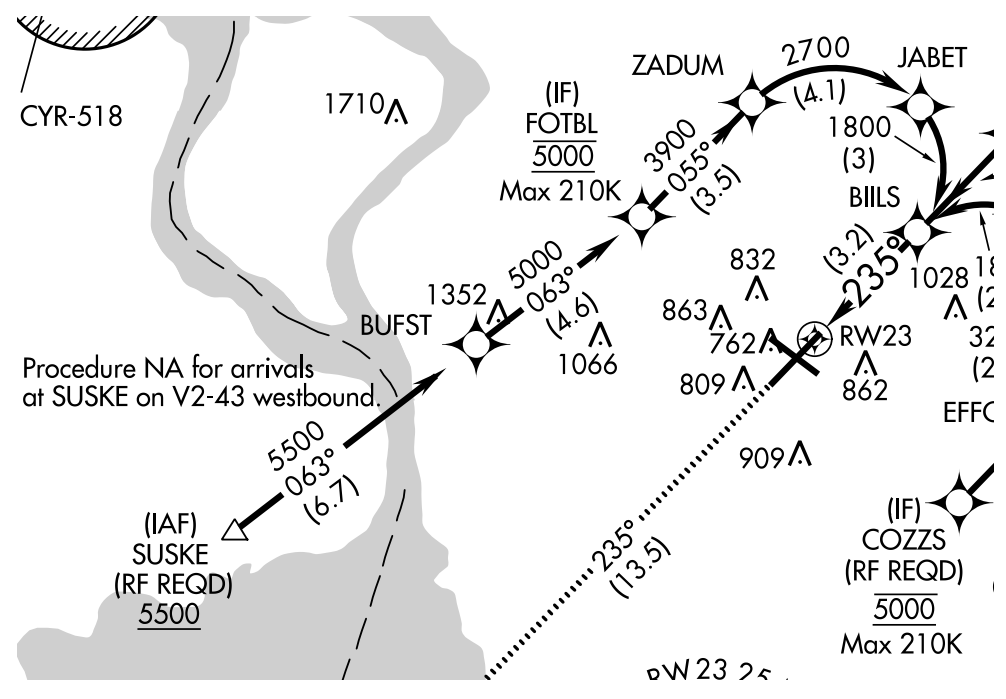

Figure 6. Approach flight plan

flight. For this flight segment, consider policies for MDP states $s=\left[\bar{V}, C_{1}, M\right] \in \mathcal{S}$ where $C_{1}=\left[\bar{\alpha}_{1}, \bar{\theta}_{1}, \bar{\phi}_{1}, \bar{h}_{1}, \bar{t}_{2}, \bar{i}_{4}, \bar{f}_{2}, P_{s}\right]$ represents features presumed to remain constant. As an example, the optimal policy action for state $s_{1}=[\bar{V}=$ $\left.\bar{v}_{3}, C_{1}, M=P\right]$ is computed by evaluating its state-action utility:

$$
\begin{gathered}
Q\left(s_{1}, N O O P\right)=\mathcal{R}\left(s_{1}, N O O P\right)+\lambda \sum_{s_{k} \in \mathcal{S}} \mathcal{T}_{P}\left(s_{k} \mid s_{1}\right) \mathcal{V}^{*}\left(s_{k}\right)=-2.55 \\
Q\left(s_{1}, T O G L\right)=\mathcal{R}\left(s_{1}, T O G L\right)+\lambda \sum_{s_{k} \in \mathcal{S}} \mathcal{T}_{E A}\left(s_{k} \mid s_{2}\right) \mathcal{V}^{*}\left(s_{k}\right)=-11.68
\end{gathered}
$$

where $s_{2}=\left[\bar{V}=\bar{v}_{3}, C_{1}, M=E A\right]$. From these calculations the optimal action in $s_{1}$ is NOOP. Table 3 illustrates the final state-action values for a subset of the other airspeed states. Note that when the flight crew has control in $\bar{V} \in\left\{\bar{v}_{3}, \bar{v}_{4}\right\}$, the policy selects NOOP. When airspeed is $\bar{V}=\bar{v}_{2}$, FSAM elects $T O G L$ to $M=E A$ because selecting $N O O P$ results in a very low state-action utility due to the relatively high likelihood of entering a high risk state $\left(\bar{V}=\bar{v}_{1}\right)$. Consequently the policy favors TOGL when $\bar{V}=\bar{v}_{2}$ because the EA controller has a higher probability of transitioning to $\bar{v}_{3}$ hence reducing risk of stall. Note that control is given back to the pilot when $\bar{V}=\bar{v}_{3}$. The policy behavior for states $s=\left[\bar{V}, C_{1}, M\right] \in \mathcal{S}$ is summarized in Fig 7 .

Table 3. State action utilities for $s=\left[\bar{V}, C_{1}, M\right]$. Left $M=P$, Right $M=E A$

\begin{tabular}{|c|c|c|}
\hline$s \in \mathcal{S}$ & $Q(s, N O O P)$ & $Q(s, T O G L)$ \\
\hline \hline$\left[\bar{v}_{1}, P\right]$ & -154.50 & -120.15 \\
\hline$\left[\bar{v}_{2}, P\right]$ & -35.63 & -14.46 \\
\hline$\left[\bar{v}_{3}, P\right]$ & -2.60 & -12.60 \\
\hline$\left[\bar{v}_{4}, P\right]$ & -1.73 & -11.73 \\
\hline
\end{tabular}

\begin{tabular}{|c|c|c|}
\hline$s \in \mathcal{S}$ & $Q(s$, NOOP $)$ & $Q(s$, TOGL $)$ \\
\hline \hline$\left[\bar{v}_{1}, E A\right]$ & -120.15 & -154.50 \\
\hline$\left[\bar{v}_{2}, E A\right]$ & -14.46 & -35.63 \\
\hline$\left[\bar{v}_{3}, E A\right]$ & -12.60 & -2.60 \\
\hline$\left[\bar{v}_{4}, E A\right]$ & -11.73 & -1.73 \\
\hline
\end{tabular}

Now consider the case where the aircraft is at ZADUM and is initiating a descending right turn at a constant airspeed. Let $C_{2}=\left[\bar{\alpha}_{1}, \bar{\theta}_{1}, \bar{h}_{4}, \bar{t}_{2}, \bar{i}_{4}, \bar{f}_{17}, P_{s}\right]$ describe the constant state features during this stage. The final state-action utilities in this flight segment are described in Table 4. In nominal conditions $\left[\bar{v}_{3}, \bar{\phi}_{1}, C_{2}, P\right]$, the policy favors NOOP. However, if the bank angle steepens $\left[\bar{v}_{3}, \bar{\phi}_{2}, C_{2}, P\right]$, the probability of stalling the aircraft is high (Appendix Tables 8 and 9) so the policy indicates a TOGL to EA. With $E A$ control, the probability of transitioning to a bank angle state that reduces stall risk is higher. Control is transferred back to the pilot when a lower risk state is attained.

\section{V.B. Flight with icing conditions}

Consider the same KBUF approach flight plan but with icing conditions illustrated in Fig 8 . Fig 8 illustrates the altitude profile of the flight plan in Fig 6. For this case the aircraft arrives at SUSKE in icing $\left(n_{\text {ice }}=1\right)$ such that the predicted time of exposure for the current flight plan is greater than the critical exposure time $t_{\text {pte }}>t_{\text {critical }}$, i.e. $\bar{I}=\bar{i}_{3}$. 


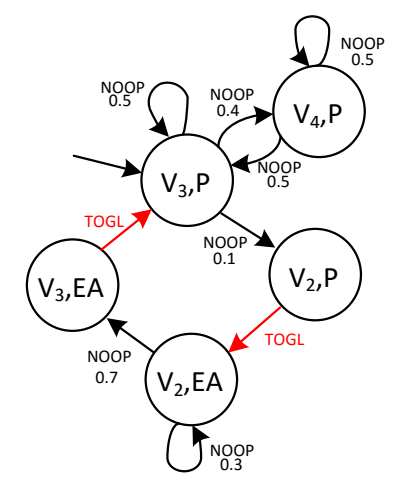

Figure 7. State transition graph for the nominal no-icing case

Table 4. State action utilities for $s=\left[\bar{V}, \bar{\Phi}, C_{2}, M\right]$. Left $M=P$, Right $M=E A$

\begin{tabular}{|c|c|c|}
\hline$s \in \mathcal{S}$ & $Q(s, N O O P)$ & $Q(s, T O G L)$ \\
\hline \hline$\left[\bar{v}_{1}, \bar{\phi}_{1}, P\right]$ & -154.50 & -120.15 \\
\hline$\left[\bar{v}_{2}, \bar{\phi}_{1}, P\right]$ & -35.99 & -14.47 \\
\hline$\left[\bar{v}_{3}, \bar{\phi}_{1}, P\right]$ & -3.19 & -12.60 \\
\hline$\left[\bar{v}_{4}, \bar{\phi}_{1}, P\right]$ & -2.38 & -11.73 \\
\hline$\left[\bar{v}_{1}, \bar{\phi}_{2}, P\right]$ & -172.52 & -120.15 \\
\hline$\left[\bar{v}_{2}, \bar{\phi}_{2}, P\right]$ & -55.67 & -14.47 \\
\hline$\left[\bar{v}_{3}, \bar{\phi}_{2}, P\right]$ & -24.16 & -12.60 \\
\hline$\left[\bar{v}_{4}, \bar{\phi}_{2}, P\right]$ & -23.55 & -11.73 \\
\hline
\end{tabular}

\begin{tabular}{|c|c|c|}
\hline$s \in \mathcal{S}$ & $Q(s, N O O P)$ & $Q(s, T O G L)$ \\
\hline \hline$\left[\bar{v}_{1}, \bar{\phi}_{1}, E A\right]$ & -120.15 & -154.50 \\
\hline$\left[\bar{v}_{2}, \bar{\phi}_{1}, E A\right]$ & -14.47 & -35.99 \\
\hline$\left[\bar{v}_{3}, \bar{\phi}_{1}, E A\right]$ & -12.60 & -3.19 \\
\hline$\left[\bar{v}_{4}, \bar{\phi}_{1}, E A\right]$ & -11.73 & -2.38 \\
\hline$\left[\bar{v}_{1}, \bar{\phi}_{2}, E A\right]$ & -120.15 & -172.52 \\
\hline$\left[\bar{v}_{2}, \bar{\phi}_{2}, E A\right]$ & -14.47 & -55.67 \\
\hline$\left[\bar{v}_{3}, \bar{\phi}_{2}, E A\right]$ & -12.60 & -24.16 \\
\hline$\left[\bar{v}_{4}, \bar{\phi}_{2}, E A\right]$ & -11.73 & -23.55 \\
\hline
\end{tabular}

Let $C_{3}=\left[\bar{\alpha}_{1}, \bar{\theta}_{1}, \bar{\phi}_{1}, \bar{h}_{4}, \bar{t}_{2}, \bar{f}_{2}, P_{s}\right]$ denote features that remain constant during this segment of the flight plan. The optimal actions at $s_{3}=\left[\bar{v}_{3}, C_{3}, \bar{i}_{3}, P\right]$ are:

$$
\begin{gathered}
Q\left(s_{3}, N O O P\right)=\mathcal{R}\left(s_{3}, N O O P\right)+\lambda \sum_{s_{k} \in \mathcal{S}} \mathcal{T}_{P}\left(s_{k} \mid s_{3}\right) \mathcal{V}^{*}\left(s_{k}\right)=-25.8 \\
Q\left(s_{3}, T O G L\right)=\mathcal{R}\left(s_{3}, T O G L\right)+\lambda \sum_{s_{k} \in \mathcal{S}} \mathcal{T}_{E A}\left(s_{k} \mid s_{4}\right) \mathcal{V}^{*}\left(s_{k}\right)=-12.83
\end{gathered}
$$

where $s_{3}=\left[\bar{v}_{3}, C_{3}, \bar{i}_{2}, P\right]$. Selecting the TOGL action results in an indirect change of the icing intensity feature from $\bar{I}=\bar{i}_{3}$ to $\bar{I}=\bar{i}_{2}$ in addition to changing the mode feature from $M=P$ to $M=E A$. The instantaneous reduction in expected icing indicated by $\bar{I}$ due to a $T O G L$ to $M=E A$ occurs due to an EA flight plan that directs the aircraft out of icing conditions quickly as illustrated in Fig 8. Switching to the Envelope-Aware controller (FMS) in this case would result in a state where $t_{\text {pte }}<=t_{\text {critical }},\left(\bar{I}=\bar{i}_{2}\right)$ since the risk of stalling is lower than in $\bar{I}=\bar{i}_{3}$ (see Appendix Tables 6 and 7). Following the new flight plan that has a lower time of exposure to icing condition minimizes the risk of in-flight icing induced stalls. Once the aircraft is out of icing conditions, the policy described in Section V.A applies and therefore control is handed back to the flight crew. Note that the reward formulation in Section IV.D does not explicitly penalize states where $\bar{I}=\bar{i}_{3}$. However, the MDP policy is able to infer $\bar{i}_{3}$ poses high risk because continuing the flight plan under icing conditions when $\bar{I}=\bar{i}_{3}$ incurs a heavy future penalty due to the higher likelihood of stalling as shown in Appendix Table 7. This work assumes that the flight crew adopts the new flight plan provided by the EA-FMS. Future work will focus on better integrating nominal FMS information into FSAM to avoid mode cycling behavior.

\section{Conclusions}

This paper presented a Markov Decision Process formulation for Flight Safety Assessment and Management that can make control authority switching decisions to avoid loss of control risk given icing conditions. A discrete state-space abstraction was designed to efficiently capture pertinent information regarding aircraft dynamics, control, 


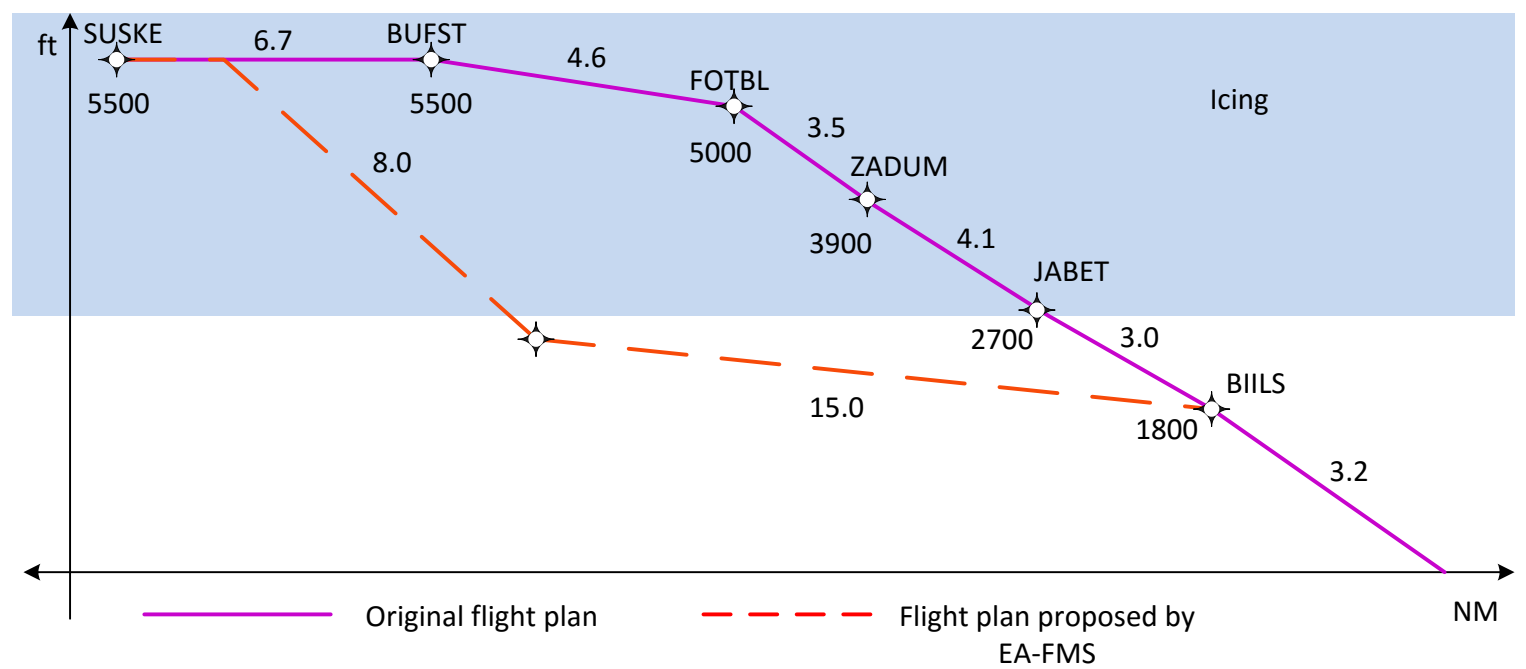

Figure 8. Vertical profile of the original flight plan indicating icing conditions and the flight plan proposed by the EA-FMS.

and expected time of exposure to icing conditions. Abstractions of these state features were constructed based on parametrized flight envelopes updated during progressive icing via online estimation. Reward functions penalized states with high loss of control risk and any control authority mode disagreeing with crew-indicated preference. Icing and no-icing case landing approach studies were presented. Several assumptions were made to simplify construction of transition probabilities. Ultimately transition probabilities and reward weights must be constructed from data collected over simulations, in-flight testing and data analysis, and focused pilot subject experiments. Ongoing work focuses on integrating the FSAM formulation presented in this paper with the other modules of EA-FMS.

\section{Appendix}

\section{State Transition Probabilities}

Consider the flight segment from SUSKE and BUFST. As indicated in Fig 6 and Fig 8, this segment requires the flight crew to maintain $5500 \mathrm{ft}$. Ideally, to express state transition probabilities in this scenario, one would require probability distributions conditioned on flight plan features of all speed ranges. However, for ease of illustration, this paper only considers distributions conditioned on $\bar{f}_{2}$ (i.e. a level-flight condition at medium airspeed). Furthermore, this paper only considers cases where the aircraft flies straight and level at a cruise power setting while maintaining angle of attack, side-slip, pitch and roll attitude within the safe operating envelopes i.e. $\bar{A}=\bar{\alpha}_{1}, \bar{\Theta}=\bar{\theta}_{1}, \bar{\Phi}=\bar{\phi}_{1}, \bar{H}=$ $\bar{h}_{1}, \bar{T}=\bar{t}_{3}$. Conditioned on these state features, the state transition probabilities for the airspeed states under pilot authority $(M=P)$ and Envelope-Aware automation authority $(M=E A)$ are indicated in Tables 5-7. Table 5 lists the airspeed transition probabilities when the aircraft is free from icing conditions (i.e. $\bar{I} \in\left\{i_{0}, i_{1}, i_{4}\right\}$ ). Table 6 lists transition probabilities for $\bar{I}=\overline{i_{2}}$ and Table 7 lists the transition probabilities for $\bar{I}=\bar{i}_{3}$. Note that with the pilot in control $M=P$, the probability of entering a high-risk airspeed state $\bar{V}=v_{1}$ and stalling increases with adverse icing conditions. However, under envelope-aware control authority $M=E A$, the probability of entering a stall state is low. Better performance when $M=E A$ is attributed to the combined EA-FMS capabilities to identify changes in dynamics, adapt controllers to these changes, estimate degraded flight envelopes and construct and follow flight plans that respect degraded envelope constraints.

Consider the flight segment from ZADUM to BIILS where the aircraft is in a descending right turn. This case considers only distributions conditioned on $\bar{f}_{17}$ (i.e. a descending turn at medium airspeed) and assumes the following states remain constant: $\bar{A}=\bar{\alpha}_{1}, \bar{\Theta}=\bar{\theta}_{1}, \bar{H}=\bar{h}_{4}, \bar{T}=\bar{t}_{2}$. For this segment, both bank angle and airspeed state are assumed likely to change. State transition probabilities for bank angle transitions under pilot $M=P$ and EnvelopeAware control $M=E A$ are indicated in Table 8 while Table 9 indicate distributions for airspeed states when $\bar{\Phi}=\bar{\phi}_{3}$. These distributions assume that $\bar{I}=\bar{i}_{0}$. Note that the probability of stalling increases when the bank angle is steep $\bar{\Phi}=\bar{\phi}_{3}$. This is attributed to the fact that during steep turns the stall speed increases. ${ }^{35}$ 


\section{Optimal Values}

The optimal values for a selected number of states relevant to the flight plan in Fig 6 are listed in Table 11. The optimal values are generated using Value Iteration ${ }^{26}$ with the reward formulation, weighting factors and transition probabilities discussed in this paper. A discount factor $\lambda=0.7$ was used. These optimal values are used in the computation of the optimal policies illustrated in Section V.

Table 5. Distribution $\mathcal{P}_{1}\left(\bar{v}_{j} \mid \bar{v}_{i}, \bar{\alpha}_{1}, \bar{\theta}_{1}, \bar{\phi}_{1,2}, \bar{h}_{1}, \bar{t}_{3}, \bar{i}_{0,1,4}, \bar{f}_{2}, P_{s}, M\right)$. Left $M=P$, Right $M=E A$

\begin{tabular}{|c|c|c|c|c|}
\hline & $\bar{v}_{1}$ & $\bar{v}_{2}$ & $\bar{v}_{3}$ & $\bar{v}_{4}$ \\
\hline $\bar{v}_{1}$ & 0.6 & 0.4 & 0.0 & 0.0 \\
\hline $\bar{v}_{2}$ & 0.4 & 0.1 & 0.5 & 0.0 \\
\hline $\bar{v}_{3}$ & 0.0 & 0.1 & 0.5 & 0.4 \\
\hline $\bar{v}_{4}$ & 0.0 & 0.0 & 0.5 & 0.5 \\
\hline
\end{tabular}

\begin{tabular}{|c|c|c|c|c|}
\hline & $\bar{v}_{1}$ & $\bar{v}_{2}$ & $\bar{v}_{3}$ & $\bar{v}_{4}$ \\
\hline $\bar{v}_{1}$ & 0.0 & 1.0 & 0.0 & 0.0 \\
\hline $\bar{v}_{2}$ & 0.0 & 0.3 & 0.7 & 0.0 \\
\hline $\bar{v}_{3}$ & 0.0 & 0.1 & 0.5 & 0.4 \\
\hline $\bar{v}_{4}$ & 0.0 & 0.0 & 0.5 & 0.5 \\
\hline
\end{tabular}

Table 6. Distribution $\mathcal{P}_{1}\left(\bar{v}_{j} \mid \bar{v}_{i}, \bar{\alpha}_{1}, \bar{\theta}_{1}, \bar{\phi}_{1,2}, \bar{h}_{1}, \bar{t}_{3}, \bar{i}_{2}, \bar{f}_{2}, P_{s}, M\right)$. Left $M=P$, Right $M=E A$

\begin{tabular}{|c|c|c|c|c|}
\hline & $\bar{v}_{1}$ & $\bar{v}_{2}$ & $\bar{v}_{3}$ & $\bar{v}_{4}$ \\
\hline $\bar{v}_{1}$ & 0.7 & 0.3 & 0.0 & 0.0 \\
\hline $\bar{v}_{2}$ & 0.5 & 0.2 & 0.3 & 0.0 \\
\hline $\bar{v}_{3}$ & 0.0 & 0.2 & 0.5 & 0.3 \\
\hline $\bar{v}_{4}$ & 0.0 & 0.0 & 0.5 & 0.5 \\
\hline
\end{tabular}

\begin{tabular}{|l|c|c|c|c|}
\hline & $\bar{v}_{1}$ & $\bar{v}_{2}$ & $\bar{v}_{3}$ & $\bar{v}_{4}$ \\
\hline $\bar{v}_{1}$ & 0.1 & 0.9 & 0.0 & 0.0 \\
\hline $\bar{v}_{2}$ & 0.0 & 0.3 & 0.7 & 0.0 \\
\hline $\bar{v}_{3}$ & 0.0 & 0.1 & 0.5 & 0.4 \\
\hline $\bar{v}_{4}$ & 0.0 & 0.0 & 0.5 & 0.5 \\
\hline
\end{tabular}

Table 7. Distribution $\mathcal{P}_{1}\left(\bar{v}_{j} \mid \bar{v}_{i}, \bar{\alpha}_{1}, \bar{\theta}_{1}, \bar{\phi}_{1,2}, \bar{h}_{1}, \bar{t}_{3}, \bar{i}_{3}, \bar{f}_{2}, P_{s}, M\right)$. Left $M=P$, Right $M=E A$

\begin{tabular}{|c|c|c|c|c|}
\hline & $\bar{v}_{1}$ & $\bar{v}_{2}$ & $\bar{v}_{3}$ & $\bar{v}_{4}$ \\
\hline $\bar{v}_{1}$ & 0.98 & 0.02 & 0.00 & 0.00 \\
\hline $\bar{v}_{2}$ & 0.85 & 0.15 & 0.00 & 0.00 \\
\hline $\bar{v}_{3}$ & 0.00 & 0.90 & 0.10 & 0.00 \\
\hline $\bar{v}_{4}$ & 0.00 & 0.00 & 0.90 & 0.10 \\
\hline
\end{tabular}

\begin{tabular}{|c|c|c|c|c|}
\hline & $\bar{v}_{1}$ & $\bar{v}_{2}$ & $\bar{v}_{3}$ & $\bar{v}_{4}$ \\
\hline $\bar{v}_{1}$ & 0.4 & 0.6 & 0.0 & 0.0 \\
\hline $\bar{v}_{2}$ & 0.2 & 0.5 & 0.3 & 0.0 \\
\hline $\bar{v}_{3}$ & 0.0 & 0.8 & 0.2 & 0.0 \\
\hline $\bar{v}_{4}$ & 0.0 & 0.0 & 0.6 & 0.4 \\
\hline
\end{tabular}

Table 8. Distribution $\mathcal{P}_{4}\left(\bar{\phi}_{j} \mid \bar{\phi}_{i}, \bar{v}_{1,2,3,4}, \bar{\alpha}_{1}, \bar{\theta}_{1}, \bar{h}_{4}, \bar{t}_{2}, \bar{i}_{4}, \bar{f}_{17}, P_{s}, M\right)$. Left $M=P$, Right $M=E A$

\begin{tabular}{|c|c|c|c|}
\hline & $\bar{\phi}_{1}$ & $\bar{\phi}_{2}$ & $\bar{\phi}_{3}$ \\
\hline $\bar{\phi}_{1}$ & 0.80 & 0.20 & 0.00 \\
\hline $\bar{\phi}_{2}$ & 0.20 & 0.30 & 0.50 \\
\hline $\bar{\phi}_{3}$ & 0.00 & 0.01 & 0.99 \\
\hline
\end{tabular}

\begin{tabular}{|c|c|c|c|}
\hline & $\bar{\phi}_{1}$ & $\bar{\phi}_{2}$ & $\bar{\phi}_{3}$ \\
\hline $\bar{\phi}_{1}$ & 1.0 & 0.0 & 0.0 \\
\hline $\bar{\phi}_{2}$ & 1.0 & 0.0 & 0.0 \\
\hline $\bar{\phi}_{3}$ & 0.0 & 1.0 & 0.0 \\
\hline
\end{tabular}

Table 9. Distribution $\mathcal{P}_{1}\left(\bar{v}_{j} \mid \bar{v}_{i}, \bar{\alpha}_{1}, \bar{\theta}_{1}, \bar{\phi}_{3}, \bar{h}_{4}, \bar{t}_{2}, \bar{i}_{4}, \bar{f}_{17}, P_{s}, M\right)$. Left $M=P$, Right $M=E A$

\begin{tabular}{|c|c|c|c|c|}
\hline & $\bar{v}_{1}$ & $\bar{v}_{2}$ & $\bar{v}_{3}$ & $\bar{v}_{4}$ \\
\hline $\bar{v}_{1}$ & 0.9 & 0.1 & 0.0 & 0.0 \\
\hline $\bar{v}_{2}$ & 0.7 & 0.2 & 0.1 & 0.0 \\
\hline $\bar{v}_{3}$ & 0.0 & 0.8 & 0.2 & 0.0 \\
\hline $\bar{v}_{4}$ & 0.0 & 0.0 & 1.0 & 0.0 \\
\hline
\end{tabular}

\begin{tabular}{|c|c|c|c|c|}
\hline & $\bar{v}_{1}$ & $\bar{v}_{2}$ & $\bar{v}_{3}$ & $\bar{v}_{4}$ \\
\hline $\bar{v}_{1}$ & 0.0 & 1.0 & 0.0 & 0.0 \\
\hline $\bar{v}_{2}$ & 0.0 & 0.6 & 0.4 & 0.0 \\
\hline $\bar{v}_{3}$ & 0.0 & 0.6 & 0.4 & 0.0 \\
\hline $\bar{v}_{4}$ & 0.0 & 0.0 & 1.0 & 0.0 \\
\hline
\end{tabular}

Table 10. Distributions $\mathcal{P}_{7}$ and $\mathcal{P}_{8}$ for $M \in\{P, E A\}$

\begin{tabular}{|c|c|c|}
\hline & $\bar{f}_{2}$ & $\bar{f}_{17}$ \\
\hline $\bar{f}_{2}$ & 0.5 & 0.5 \\
\hline $\bar{f}_{17}$ & 0.5 & 0.5 \\
\hline
\end{tabular}

\begin{tabular}{|c|c|c|c|}
\hline & $\bar{i}_{2}$ & $\bar{i}_{3}$ & $\bar{i}_{4}$ \\
\hline $\bar{i}_{2}$ & 0.5 & 0.0 & 0.5 \\
\hline $\bar{i}_{3}$ & 0.0 & 0.7 & 0.3 \\
\hline $\bar{i}_{4}$ & 0.0 & 0.0 & 1.0 \\
\hline
\end{tabular}


Table 11. Optimal values for relevant states with $\bar{S}=P_{S}$

\begin{tabular}{|c|c|c|c|c|c|c|c|c|c|c|}
\hline & $M$ & $\bar{I}$ & $\bar{F}$ & $\bar{T}$ & $\bar{H}$ & $\bar{\Phi}$ & $\bar{\Theta}$ & $\bar{A}$ & $\bar{V}$ & $\mathcal{V}^{*}(s)$ \\
\hline 1 & $P$ & $\bar{i}_{4}$ & $\bar{f}_{2}$ & $\overline{t_{3}}$ & $\bar{h}_{1}$ & $\bar{\phi}_{1}$ & $\bar{\theta}_{1}$ & $\bar{\alpha}_{1}$ & $\bar{v}_{1}$ & -120.1 \\
\hline 2 & $P$ & $\bar{i}_{4}$ & $\bar{f}_{2}$ & $\bar{t}_{3}$ & $\bar{h}_{1}$ & $\bar{\phi}_{1}$ & $\bar{\theta}_{1}$ & $\bar{\alpha}_{1}$ & $\bar{v}_{2}$ & -14.5 \\
\hline 3 & $P$ & $\bar{i}_{4}$ & $\bar{f}_{2}$ & $\bar{t}_{3}$ & $\bar{h}_{1}$ & $\bar{\phi}_{1}$ & $\bar{\theta}_{1}$ & $\bar{\alpha}_{1}$ & $\bar{v}_{3}$ & -2.6 \\
\hline 4 & $P$ & $\bar{i}_{4}$ & $\bar{f}_{2}$ & $\bar{t}_{3}$ & $\bar{h}_{1}$ & $\bar{\phi}_{1}$ & $\bar{\theta}_{1}$ & $\bar{\alpha}_{1}$ & $\bar{v}_{4}$ & -1.7 \\
\hline 5 & $P$ & $\overline{\bar{i}}_{4}$ & $\bar{f}_{17}$ & $\overline{t_{2}}$ & $\bar{h}_{4}$ & $\bar{\phi}_{1}$ & $\bar{\theta}_{1}$ & $\bar{\alpha}_{1}$ & $\bar{v}_{1}$ & -120.1 \\
\hline 6 & $P$ & $\overline{\bar{i}}_{4}$ & $\bar{f}_{17}$ & $\overline{t_{2}}$ & $\bar{h}_{4}$ & $\bar{\phi}_{1}$ & $\bar{\theta}_{1}$ & $\bar{\alpha}_{1}$ & $\bar{v}_{2}$ & -14.5 \\
\hline 7 & $P$ & $\bar{i}_{4}$ & $\bar{f}_{17}$ & $\overline{t_{2}}$ & $\bar{h}_{4}$ & $\bar{\phi}_{1}$ & $\bar{\theta}_{1}$ & $\bar{\alpha}_{1}$ & $\bar{v}_{3}$ & -3.2 \\
\hline 8 & $P$ & $\bar{i}_{4}$ & $\bar{f}_{17}$ & $\overline{t_{2}}$ & $\bar{h}_{4}$ & $\bar{\phi}_{1}$ & $\bar{\theta}_{1}$ & $\bar{\alpha}_{1}$ & $\bar{v}_{4}$ & -2.4 \\
\hline 9 & $P$ & $\bar{i}_{4}$ & $\bar{f}_{17}$ & $\bar{t}_{2}$ & $\bar{h}_{4}$ & $\bar{\phi}_{2}$ & $\bar{\theta}_{1}$ & $\bar{\alpha}_{1}$ & $\bar{v}_{1}$ & -120.1 \\
\hline 10 & $P$ & $\bar{i}_{4}$ & $\bar{f}_{17}$ & $\overline{t_{2}}$ & $\bar{h}_{4}$ & $\bar{\phi}_{2}$ & $\bar{\theta}_{1}$ & $\bar{\alpha}_{1}$ & $\bar{v}_{2}$ & -14.5 \\
\hline 11 & $P$ & $\bar{i}_{4}$ & $\bar{f}_{17}$ & $\overline{t_{2}}$ & $\bar{h}_{4}$ & $\bar{\phi}_{2}$ & $\bar{\theta}_{1}$ & $\bar{\alpha}_{1}$ & $\bar{v}_{3}$ & -12.6 \\
\hline 12 & $P$ & $\bar{i}_{4}$ & $\bar{f}_{17}$ & $\overline{t_{2}}$ & $\bar{h}_{4}$ & $\bar{\phi}_{2}$ & $\bar{\theta}_{1}$ & $\bar{\alpha}_{1}$ & $\bar{v}_{4}$ & -11.7 \\
\hline 13 & $P$ & $\bar{i}_{3}$ & $\bar{f}_{2}$ & $\overline{t_{3}}$ & $\bar{h}_{1}$ & $\bar{\phi}_{1}$ & $\bar{\theta}_{1}$ & $\bar{\alpha}_{1}$ & $\bar{v}_{1}$ & -169.6 \\
\hline 14 & $P$ & $\bar{i}_{3}$ & $\bar{f}_{2}$ & $\overline{t_{3}}$ & $\bar{h}_{1}$ & $\bar{\phi}_{1}$ & $\bar{\theta}_{1}$ & $\bar{\alpha}_{1}$ & $\bar{v}_{2}$ & -49.2 \\
\hline 15 & $P$ & $\bar{i}_{3}$ & $\bar{f}_{2}$ & $\bar{t}_{3}$ & $\bar{h}_{1}$ & $\bar{\phi}_{1}$ & $\bar{\theta}_{1}$ & $\bar{\alpha}_{1}$ & $\bar{v}_{3}$ & -25.8 \\
\hline 16 & $P$ & $\bar{i}_{3}$ & $\overline{f_{2}}$ & $\bar{t}_{3}$ & $\bar{h}_{1}$ & $\bar{\phi}_{1}$ & $\bar{\theta}_{1}$ & $\bar{\alpha}_{1}$ & $\bar{v}_{4}$ & -12.6 \\
\hline 17 & $E A$ & $\bar{i}_{4}$ & $\bar{f}_{2}$ & $\overline{t_{3}}$ & $\bar{h}_{1}$ & $\bar{\phi}_{1}$ & $\bar{\theta}_{1}$ & $\bar{\alpha}_{1}$ & $\bar{v}_{1}$ & -120.1 \\
\hline 18 & $E A$ & $\bar{i}_{4}$ & $\bar{f}_{2}$ & $\overline{t_{3}}$ & $\bar{h}_{1}$ & $\bar{\phi}_{1}$ & $\bar{\theta}_{1}$ & $\bar{\alpha}_{1}$ & $\bar{v}_{2}$ & -14.5 \\
\hline 19 & $E A$ & $\bar{i}_{4}$ & $\bar{f}_{2}$ & $\bar{t}_{3}$ & $\bar{h}_{1}$ & $\bar{\phi}_{1}$ & $\bar{\theta}_{1}$ & $\bar{\alpha}_{1}$ & $\bar{v}_{3}$ & -2.6 \\
\hline 20 & $E A$ & $\bar{i}_{4}$ & $\bar{f}_{2}$ & $\overline{t_{3}}$ & $\bar{h}_{1}$ & $\bar{\phi}_{1}$ & $\bar{\theta}_{1}$ & $\bar{\alpha}_{1}$ & $\bar{v}_{4}$ & -1.7 \\
\hline 21 & $E A$ & $\bar{i}_{4}$ & $\bar{f}_{17}$ & $\overline{t_{2}}$ & $\bar{h}_{4}$ & $\bar{\phi}_{1}$ & $\bar{\theta}_{1}$ & $\bar{\alpha}_{1}$ & $\bar{v}_{1}$ & -120.1 \\
\hline 22 & $E A$ & $\overline{\bar{i}}_{4}$ & $\bar{f}_{17}$ & $\overline{t_{2}}$ & $\bar{h}_{4}$ & $\bar{\phi}_{1}$ & $\bar{\theta}_{1}$ & $\bar{\alpha}_{1}$ & $\bar{v}_{2}$ & -14.5 \\
\hline 23 & $E A$ & $\bar{i}_{4}$ & $\bar{f}_{17}$ & $\overline{t_{2}}$ & $\bar{h}_{4}$ & $\bar{\phi}_{1}$ & $\bar{\theta}_{1}$ & $\bar{\alpha}_{1}$ & $\bar{v}_{3}$ & -3.2 \\
\hline 24 & $E A$ & $\bar{i}_{4}$ & $\bar{f}_{17}$ & $\overline{t_{2}}$ & $\bar{h}_{4}$ & $\bar{\phi}_{1}$ & $\bar{\theta}_{1}$ & $\bar{\alpha}_{1}$ & $\bar{v}_{4}$ & -2.4 \\
\hline 25 & $E A$ & $\bar{i}_{4}$ & $\bar{f}_{17}$ & $\overline{t_{2}}$ & $\bar{h}_{4}$ & $\bar{\phi}_{2}$ & $\bar{\theta}_{1}$ & $\bar{\alpha}_{1}$ & $\bar{v}_{1}$ & -120.1 \\
\hline 26 & $E A$ & $\bar{i}_{4}$ & $\bar{f}_{17}$ & $\overline{t_{2}}$ & $\bar{h}_{4}$ & $\bar{\phi}_{2}$ & $\bar{\theta}_{1}$ & $\bar{\alpha}_{1}$ & $\bar{v}_{2}$ & -14.5 \\
\hline 27 & $E A$ & $\bar{i}_{4}$ & $\bar{f}_{17}$ & $\overline{t_{2}}$ & $\bar{h}_{4}$ & $\bar{\phi}_{2}$ & $\bar{\theta}_{1}$ & $\bar{\alpha}_{1}$ & $\bar{v}_{3}$ & -12.6 \\
\hline 28 & $E A$ & $\bar{i}_{4}$ & $\bar{f}_{17}$ & $\overline{t_{2}}$ & $\bar{h}_{4}$ & $\bar{\phi}_{2}$ & $\bar{\theta}_{1}$ & $\bar{\alpha}_{1}$ & $\bar{v}_{4}$ & -11.7 \\
\hline 29 & $E A$ & $\bar{i}_{2}$ & $\bar{f}_{2}$ & $\overline{t_{3}}$ & $\bar{h}_{1}$ & $\bar{\phi}_{1}$ & $\bar{\theta}_{1}$ & $\bar{\alpha}_{1}$ & $\bar{v}_{1}$ & -127.9 \\
\hline 30 & $E A$ & $\bar{i}_{2}$ & $\bar{f}_{2}$ & $\overline{t_{3}}$ & $\bar{h}_{1}$ & $\bar{\phi}_{1}$ & $\bar{\theta}_{1}$ & $\bar{\alpha}_{1}$ & $\bar{v}_{2}$ & -14.7 \\
\hline 31 & $E A$ & $\bar{i}_{2}$ & $\bar{f}_{2}$ & $\bar{t}_{3}$ & $\bar{h}_{1}$ & $\bar{\phi}_{1}$ & $\bar{\theta}_{1}$ & $\bar{\alpha}_{1}$ & $\bar{v}_{3}$ & -3.7 \\
\hline 32 & $E A$ & $\overline{\bar{i}_{2}}$ & $\bar{f}_{2}$ & $\overline{t_{3}}$ & $\bar{h}_{1}$ & $\bar{\phi}_{1}$ & $\bar{\theta}_{1}$ & $\bar{\alpha}_{1}$ & $\bar{v}_{4}$ & -2.0 \\
\hline
\end{tabular}

\section{Acknowledgments}

This work was supported in part by the National Aeronautics and Space Administration under Cooperative Agreement NNX12AM54A. The authors would like to thank Pedro Di Donato for suggestions regarding flight planner state abstractions.

\section{References}

\footnotetext{
${ }^{1}$ Belcastro, C. M. and Foster, J. V., "Aircraft Loss of Control Accident Analysis," Proc. AIAA Guidance Navigation, and Control Conference, Toronto, Ontario, 2010.

${ }^{2}$ Sand, W. R., Cooper, W. A., Politovich, M. K., and Veal, D. L., "Icing conditions encountered by a research aircraft," Journal of climate and applied meteorology, Vol. 23, No. 10, 1984, pp. 1427-1440.

${ }^{3}$ Van Dyke, D., “Tailplane icing: survival knowledge for pilots," Professional Pilot, Vol. 43, No. 9, 2009.
}

15 of 16 
${ }^{4}$ Bureau d'Enquetes et d'Analyses, "Final report on the accident on 1st June 2009 to the Airbus A330-203 registered F-GZCP operated by Air France flight AF 447 Rio de Janeiro-Paris," Ministère de l'Écologie. du Dévéloppement durable, des Transports et du Logement, Paris, 2012.

${ }^{5}$ Dutch Safety Board, "Crashed during approach, Boeing 737-800, near Amsterdam Schiphol airport," Tech. rep., Technical report, Dutch Safety Board. Available at http://www.onderzoeksraad.nl/docs/rapporten/Rapport \_TA \_ENG\_web.pdf, 2010.

${ }^{6}$ Balachandran, S. and Atkins, E. M., "Flight Safety Assessment and Management during Takeoff," AIAA Infotech@Aerospace Conference, Boston, MA, 2013.

${ }^{7}$ Balachandran, S. and Atkins, E. M., "An Evaluation of Flight Safety Assessment and Management to avoid Loss of Control during Takeoff," AIAA Guidance, Navigation and Control Conference, National Harbor, MD, 2014.

${ }^{8}$ Balachandran, S. and Atkins, E. M., "Flight Safety Assessment and Management for Takeoff using Deterministic Moore Machines," Journal of Aerospace Information Systems, Vol. 12, No. 9, Nov 2015, pp. 599-615, doi:10.2514/1.I010350.

${ }^{9}$ McDonough, K., Kolmanovsky, I., and Atkins, E. M., "Recoverable Sets of Initial Conditions and Their Use for Aircraft Flight Planning After a Loss of Control Event," Proc. AIAA Guidance Navigation, and Control Conference, National Harbor, Maryland, 2014.

${ }^{10}$ McDonough, K. and Kolmanovsky, I., "Integrator Resetting for Enforcing Constraints in Aircraft Flight Control Systems," Proc. AIAA Guidance Navigation, and Control Conference, Kissimmee, Florida, 2015.

${ }^{11}$ Di Donato, P. F. A. and Atkins, E. M., "An Off-Runway Emergency Landing Aid for a Small Aircraft Experiencing Loss of Thrust," AIAA Infotech@Aerospace Conference, Kissimmee, FL, 2015.

${ }^{12}$ Yi, G., Zhong, J., Atkins, E. M., and Wang, C., “Trim State Discovery with Physical Constraints,” Journal of Aircraft, 2014 , pp. 1-17.

${ }^{13}$ Yu, M. J., Zhong, J., Atkins, E. M., Kolmanovsky, I., and Bernstein, D. S., "Trim-commanded adaptive control for waypoint-defined trajectory following," Proc. AIAA Guidance Navigation, and Control Conference, Boston, MA, 2013.

${ }^{14}$ Yu, M.-J., McDonough, K., Bernstein, D. S., and Kolmanovsky, I., "Retrospective Cost Model Refinement for Aircraft Fault Signature Detection," American Control Conference (ACC), 2014, IEEE, 2014, pp. 2486-2491, doi:10.1109/ACC.2014.6858876.

${ }^{15}$ Schuet, S., Lombaerts, T., Acosta, D., Wheeler, K., and Kaneshige, J., "An Adaptive Nonlinear Aircraft Maneuvering Envelope Estimation Approach for Online Applications," AIAA Guidance, Navigation and Control Conference, Jan.

${ }^{16}$ Santillo, M. A. and Bernstein, D. S., "Adaptive control based on retrospective cost optimization," Journal of guidance, control, and dynamics, Vol. 33, No. 2, 2010, pp. 289-304.

${ }^{17}$ Gregory, I. M., Cao, C., Xargay, E., Hovakimyan, N., and Zou, X., "L1 Adaptive Control Design for NASA AirSTAR Flight Test Vehicle," AIAA guidance, navigation, and control conference, No. AIAA 2010-8142, 2009.

${ }^{18}$ Di Donato, P. F. A., Balachandran, S., McDonough, K., Atkins, E., and Kolmanovsky, I., "Envelope Aware Flight Management for Loss of Control Prevention given Rudder Jam," Submitted to the Journal of Guidance, Navigation and Control, 2015.

${ }^{19}$ Bragg, M. B., Basar, T., Perkins, W. R., Selig, M. S., Voulgaris, P. G., Melody, J. W., and Sarter, N. B., "Smart icing systems for aircraft icing safety,", No. AIAA-2002-0813, 2002, 10.2514/6.2002-813.

${ }^{20}$ Schuchard, E. A., Melody, J. W., Basar, T., Perkins, W. R., and Voulgaris, P., "Detection and classification of aircraft icing using neural networks," 38th AIAA Aerospace Sciences Meeting and Exhibit, 2000.

${ }^{21}$ Sharma, V., Voulgaris, P. G., and Frazzoli, E., "Aircraft autopilot analysis and envelope protection for operation under icing conditions," Journal of guidance, control, and dynamics, Vol. 27, No. 3, 2004, pp. 454-465.

${ }^{22}$ Sarter, N. B. and Schroeder, B., "Supporting decision making and action selection under time pressure and uncertainty: The case of in-flight icing," Human Factors: The Journal of the Human Factors and Ergonomics Society, Vol. 43, No. 4, 2001, pp. 573-583.

${ }^{23}$ Gingras, D. R., Barnhart, B., Ranaudo, R., Ratvasky, T. P., and Morelli, E., "Envelope Protection for In-Flight Ice Contamination," 47th AIAA Aerospace Sciences Meeting, Orlando, FL.

${ }^{24}$ Lombaerts, T., Schuet, S., Acosta, D., Kaneshige, J., Shish, K., and Martin, L., "Piloted Simulator Evaluation of Maneuvering Envelope Information for Flight Crew Awareness," AIAA Guidance, Navigation, and Control Conference, Kissimmee, FL, Jan.

${ }^{25}$ Lombaerts, T., Schuet, S. R., Wheeler, K. R., Acosta, D. M., and Kaneshige, J. T., "Safe maneuvering envelope estimation based on a physical approach," AIAA Guidance, Navigation, and Control Conference, Boston, MA, August.

${ }^{26}$ Puterman, M. L., Markov Decision Process: Discrete Stochastic Dynamic Programming, chap. 2-6, John Wiley \& Sons, Inc, 1994.

${ }^{27}$ Russell, S. J. and Norvig, P., Artificial intelligence: A Modern Approach, chap. 17, Pearson Education Limited, 2014.

${ }^{28}$ Balachandran, S. and Atkins, E. M., "A Markov Decision Process Framework for Flight Safety Assessment and Management," Submitted to the Journal of Guidance, Navigation and Control, 2015.

${ }^{29}$ Airspeed Indicator, Image source:https://commons .wikimedia.org/wiki/File:Airspeed_Indicator.svg[accessed Dec'15].

${ }^{30}$ Vertical Speed Indicator, Image source:https://commons.wikimedia.org/wiki/File:Vertical_Speed_Indicator.svg[accessed Dec'15].

${ }^{31}$ Jason Tatum, RPM gauge, Image Source: http://siminnovations.com/online_store/instruments/preview/ 32f9f183-506a-485d-b89b-716942245354.png[accessed Dec'15].

${ }^{32}$ Wilborn, J. E. and Foster, J. V., "Defining Commercial Transport Loss-of-Control: A Quantitative Approach," Proc. AIAA Atmospheric Flight Mechanics Conference and Exhibit, Providence, Rhode Island, 2004

${ }^{33}$ Politovich, M. K., "Response of a research aircraft to icing and evaluation of severity indices," Journal of aircraft, Vol. 33, No. 2, 1996, pp. 291-297.

34"Loss of Control on Approach Colgan Air, Inc. Operating as Continental Connection Flight 3407 Bombardier DHC-8-400, N200WQ," Accident report NTSB/AAR-10/01, 2010.

${ }^{35}$ Stevens, L. B. and Lewis, L. F., Aircraft Control and Simulation, chap. 1-2, Hoboken, NJ: Wiley, 2003, pp. 1-137. 